\title{
Enhanced lipid production by Yarrowia lipolytica cultured with synthetic and waste-derived high-content volatile fatty acids under alkaline conditions
}

\author{
Ruiling Gao, Zifu Li ${ }^{*} \mathbb{D}$, Xiaoqin Zhou, Wenjun Bao, Shikun Cheng and Lei Zheng
}

\begin{abstract}
Background: Volatile fatty acids (VFAs) can be effective and promising alternate carbon sources for microbial lipid production by a few oleaginous yeasts. However, the severe inhibitory effect of high-content ( $>10 \mathrm{~g} / \mathrm{L}$ ) VFAs on these yeasts has impeded the production of high lipid yields and their large-scale application. Slightly acidic conditions have been commonly adopted because they have been considered favorable to oleaginous yeast cultivation. However, the acidic pH environment further aggravates this inhibition because VFAs appear largely in an undissociated form under this condition. Alkaline conditions likely alleviate the severe inhibition of high-content VFAs by significantly increasing the dissociation degree of VFAs. This hypothesis should be verified through a systematic research.

Results: The combined effects of high acetic acid concentrations and alkaline conditions on VFA utilization, cell growth, and lipid accumulation of Yarrowia lipolytica were systematically investigated through batch cultures of $Y$. lipolytica by using high concentrations (30-110 g/L) of acetic acid as a carbon source at an initial pH ranging from 6 to 10. An initial pH of 8 was determined as optimal. The highest biomass and lipid production (37.14 and $10.11 \mathrm{~g} / \mathrm{L}$ ) were obtained with $70 \mathrm{~g} / \mathrm{L}$ acetic acid, whereas cultures with $>70 \mathrm{~g} / \mathrm{L}$ acetic acid had decreased biomass and lipid yield due to excessive anion accumulation. Feasibilities on high-content propionic acid, butyric acid, and mixed VFAs were compared and evaluated. Results indicated that $Y_{X / S}$ and $Y_{L / S}$ of cultures on butyric acid $(0.570,0.144)$ were comparable with those on acetic acid $(0.578,0.160)$ under alkaline conditions. The performance on propionic acid was much inferior to that on other acids. Mixed VFAs were more beneficial to fast adaptation and lipid production than single types of VFA. Furthermore, cultures on food waste (FW) and fruit and vegetable waste (FVW) fermentate were carried out and lipid production was effectively improved under this alkaline condition. The highest biomass and lipid production on FW fermentate reached $14.65 \mathrm{~g} / \mathrm{L}\left(Y_{X / S}: 0.414\right)$ and $3.20 \mathrm{~g} / \mathrm{L}\left(Y_{L / S}: 0.091\right)$ with a lipid content of $21.86 \%$, respectively. By comparison, the highest biomass and lipid production on FVW fermentate were $11.84 \mathrm{~g} / \mathrm{L}\left(Y_{X / S}: 0.534\right)$ and $3.08 \mathrm{~g} / \mathrm{L}\left(Y_{L / S}: 0.139\right)$, respectively, with a lipid content of $26.02 \%$.
\end{abstract}

Conclusions: This study assumed and verified that alkaline conditions (optimal pH 8) could effectively alleviate the lethal effect of high-content VFA on Y. lipolytica and significantly improve biomass and lipid production. These results could provide a new cultivation strategy to achieve simple utilizations of high-content VFAs and increase lipid

\footnotetext{
*Correspondence: zifulee@aliyun.com

School of Energy and Environmental Engineering, Beijing Key Laboratory

of Resource-oriented Treatment of Industrial Pollutants, University

of Science and Technology Beijing, Beijing 100083, People's Republic

of China
}

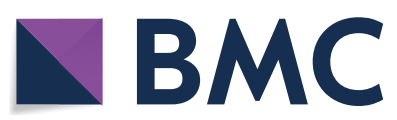

(c) The Author(s) 2020. This article is licensed under a Creative Commons Attribution 4.0 International License, which permits use, sharing, adaptation, distribution and reproduction in any medium or format, as long as you give appropriate credit to the original author(s) and the source, provide a link to the Creative Commons licence, and indicate if changes were made. The images or other third party material in this article are included in the article's Creative Commons licence, unless indicated otherwise in a credit line to the material. If material is not included in the article's Creative Commons licence and your intended use is not permitted by statutory regulation or exceeds the permitted use, you will need to obtain permission directly from the copyright holder. To view a copy of this licence, visit http://creativeco mmons.org/licenses/by/4.0/. The Creative Commons Public Domain Dedication waiver (http://creativecommons.org/publicdomain/ zero/1.0/) applies to the data made available in this article, unless otherwise stated in a credit line to the data. 
production. Feasibilities on FW and FVW-derived VFAs were evaluated, and meaningful information was provided for practical applications.

Keywords: Microbial lipids, Yarrowia lipolytica, High-content volatile fatty acids, Alkaline conditions

\section{Background}

Lipids synthesized by oleaginous yeasts are highly promising feedstock for biodiesel production primarily because of their high potential productivity and short production period [1]. However, their large-scale application is limited by their high production costs, which mainly stem from traditional but expensive fermentation substrates, such as glucose [2]. Therefore, the exploitation of low-cost alternative substrates is becoming a research hot spot. Volatile fatty acids (VFAs), which refer to short chain fatty acids (C1-C6), can be cheaply produced from the anaerobic fermentation of various organic wastes [3]. Economic calculation results have shown that VFAs produced from food waste (FW) cost 30 dollars/ton, which is $<10 \%$ of the cost of a ton of glucose [4]. Besides their sufficient and cost-effective sources, VFAs also have higher theoretical conversion efficiencies and shorter metabolic pathways to lipids compared with other sugarbased carbon sources [5-7]. Therefore, VFAs have been considered as promising alternate carbon source for microbial lipids production. Recently, research on the bioconversion of VFAs into high value-added microbial lipids have attracted significant attention and some oleaginous yeast and very few molds have been proven to utilize VFAs to synthesize lipids, e.g. Yarrowia lipolytica, Cryptococcus curvatus, Cryptococcus albidus [8].

VFAs can be routinely generated during the acidogenic fermentation of various organic wastes. In general, VFAs derived from sewage excess sludge have a concentration of 2-8 g/L [9], whereas VFAs derived from FW, animal or human feces, and high-content organic wastewater can have a relatively high concentration of $10-40 \mathrm{~g} / \mathrm{L}$ [10]. VFA composition can be affected by substrate type and fermentation conditions [11]. Among all types of VFAs, acetic acid is generally the highest, accounting for $43-69 \%$ [12], followed by propionic acid (10-54\%) [13] and butyric acid (9-46.9\%) [8]. In recent years, these waste-derived VFAs turn out to be effective carbon sources for the synthesis of high value-added microbial lipids by oleaginous yeasts under certain culture conditions [1]. Previous studies focused on investigating the lipid production potential and suitable culture conditions of different oleaginous yeast strains by using VFAs as carbon sources. Most of these studies have used low concentrations $(2-10 \mathrm{~g} / \mathrm{L})$ of acetic acid or VFA mixtures and have prioritized acetic acid as the carbon source [14]. On the one hand, acetic acid is considered superior to other acids for lipid synthesis because of its relatively shorter conversion pathway to produce acetyl-CoA, an important precursor in lipid biosynthesis. On the other hand, high VFA concentrations adversely affect yeast cell growth, sharply reducing the utilization ratio of VFAs and lipid yield. Fei et al. [4] first reported this inhibitory effect in 2011 by using VFA mixtures with an initial concentration higher than $5 \mathrm{~g} / \mathrm{L}$ as a carbon source to cultivate $C$. albidus. Furthermore, different VFA types and concentrations can produce different intensities of inhibition [15-17]. In general, substrate inhibition on oleaginous microorganisms from high-content VFAs becomes non-negligible when the VFA concentration in culture media exceeds $10 \mathrm{~g} / \mathrm{L}$ and can even be a fatal factor to cell growth when it exceeds $20 \mathrm{~g} / \mathrm{L}[3,17]$. In previous studies, at appropriate culture conditions, $<2 \mathrm{~g} / \mathrm{L}$ lipid concentration and $10-40 \%$ lipid content were commonly obtained in batch cultivation by using low concentrations $(2-10 \mathrm{~g} / \mathrm{L})$ of VFAs as a carbon source [17]. To promote the yield of microbial lipids from VFAs, researchers also explored different cultivation modes, such as fed-batch $[15,18]$, repeated batch [19], sequencing batch [1, 20], continuous cultivation [21], and two-staged cultivation $[16,22]$. However, the lipid yields in these studies are unsatisfactory because of the low VFA concentrations. Therefore, substrate inhibition attributed to high VFA concentrations on these oleaginous yeasts has impeded high-lipid-yield production and large-scale applications.

It is commonly accepted that the adverse effects of VFAs on yeast cells are mainly caused by the undissociated form of the acid molecules. When the lipophilic undissociated form of VFAs permeates into the plasma membrane, they dissociate intracellularly into hydrogen ions and the corresponding anions, leading to cytosolic acidification and inducing stress on cell metabolism [2325]. Yeasts possess a series of regulatory mechanisms to control the entry and exit of hydrogen ions into their cells to maintain a near neutral intracellular environment. However, this capacity is limited for adaptation and regulation [17]. Environmental $\mathrm{pH}$ has a crucial effect on the cell proliferation and lipid synthesis of oleaginous microorganisms [26]. Acidic cultivation conditions ( $\mathrm{pH} 5-6$ ) are usually preferred by oleaginous yeasts when glucose is used as a carbon source. In addition, some scholars held the view that alkaline environment might have certain adverse effects on the lipid synthesis of yeasts [4, 6 , 27]. Hence, previous researches commonly adopted 
slightly acidic conditions $(\mathrm{pH}$ 5.6-7) rather than alkaline conditions for their cultivation even with VFAs as a carbon source. Considering that the change in carbon source may lead to differences in optimal conditions, the influence of $\mathrm{pH}$ has been investigated $[4,28]$. However, the best lipid production performance of the strains still occurs under slightly acidic conditions ( $\mathrm{pH}$ 5.6-7) probably because of the low VFA concentrations and the limited adaptation and regulation capacity of strains. Fei et al. [4] studied the influence of different initial $\mathrm{pH}$ of culture media on the biomass and lipid production of $C$. albidus with $2 \mathrm{~g} / \mathrm{L}$ VFAs as a carbon source and found that the highest lipid concentration and content $(0.3 \mathrm{~g} / \mathrm{L}$ and 26.7\%) are achieved at pH 6.0. Gao et al. [17] reported that the cell growth and lipid accumulation of Yarrowia lipolytica at an initial $\mathrm{pH}$ of 6.0 are highly similar when $2.5 \mathrm{~g} / \mathrm{L}$ acetic acid, butyric acid, and propionic acid are used as sole carbon sources; this result is also comparable with that of cultures at the same glucose concentration. These results have shown that the adverse effects of VFAs at low concentrations are weak and negligible. However, when high concentrations $(>10 \mathrm{~g} / \mathrm{L})$ of VFAs are used as the sole carbon source, their adverse effects should be considered. The acidic $\mathrm{pH}$ environment further aggravates this inhibition as VFAs appear largely in an undissociated form under this condition. Liu et al. [3] showed that $C$. curvatus cultured with $30 \mathrm{~g} / \mathrm{L}$ acetic acid grows much better in an alkaline medium than it does in an acidic medium, but no further systematic research has been conducted. It could be assumed that an alkaline $\mathrm{pH}$ environment can effectively alleviate the severe effect of high-content VFA by considerably increasing the degree of dissociation of VFAs in culture media and thus help increase lipid productions. Further work is needed to test this hypothesis.

Yarrowia lipolytica is considered a model microorganism for lipid production because of its ability to accumulate high levels of lipids and its suite of efficient genetic tools [29]. Our previous study [17] demonstrated the feasibility of using low VFA concentrations for lipid productions by $Y$. lipolytica. The present study mainly aims to systematically investigate and evaluate the influence of alkaline conditions on alleviating the inhibitory effects of high-content VFAs on oleaginous yeasts for achieving a high cell density and a high lipid yield. First, the adaptable $\mathrm{pH}$ range of $Y$. lipolytica when VFAs were used as a carbon source was investigated at a low acetic acid concentration $(3 \mathrm{~g} / \mathrm{L})$. Then, the VFA utilization, cell growth, and lipid accumulation of $Y$. lipolytic were then evaluated on batch cultures by using high concentrations $(30-110 \mathrm{~g} / \mathrm{L})$ of acetic acid as the sole carbon source at initial $\mathrm{pH}$ of $6,7,8,9$, and 10 . As two other typical wastederived VFA types, propionic acid and butyric acid, along with the mixed VFAs (acetic acid: propionic acid: butyric acid $=5: 2: 3$ ), as carbon sources were also investigated and compared in terms of their feasibilities in cultures. Finally, cultures on undiluted FW and fruit and vegetable waste (FVW) fermentate were prepared to evaluate their potential for practical applications.

\section{Results and discussion \\ Evaluation of the $\mathrm{pH}$ tolerance and lipid production of $Y$. lipolytica by using low-concentration acetic acid as a carbon source}

$\mathrm{pH}$ is an important factor that affects the cell proliferation and lipid synthesis of oleaginous microorganisms [26]. Different microorganisms can adapt to different ranges of environmental $\mathrm{pH}$ [17]. The inhibitory effects of weak acids on microorganisms have been ascribed to proton motive force uncoupling and anion accumulation, and the counteracting responses of the yeast cell involve the export of protons and anions at the expense of ATP $[30,31]$. Accordingly, the inhibitory effects of VFAs on yeasts are not only concentration dependent but also related to the $\mathrm{pH}$ of the environment and to the dissociation degree of VFAs [32].

Figure 1 illustrates the effects of initial $\mathrm{pH}$ on cell growth and lipid accumulation in a batch culture of $Y$. lipolytica by using a low concentration $(3 \mathrm{~g} / \mathrm{L})$ of acetic acid as the sole carbon source. It is found that from $\mathrm{pH}$ 6 to 9, Y. lipolytica cells started proliferating without an obvious lag phase $(<3 \mathrm{~h})$ and showed good performance on biomass and lipid production. The highest lipid yield $(0.91 \mathrm{~g} / \mathrm{L})$ was obtained at $\mathrm{pH} 7$, followed by cultures at $\mathrm{pH} 6$ with a comparable lipid yield of $0.90 \mathrm{~g} / \mathrm{L}$. Specifically, the highest biomass production $(2.83 \mathrm{~g} / \mathrm{L})$ was obtained at $\mathrm{pH} 7$, and the highest lipid content (32.62\%) was obtained at $\mathrm{pH} 6$. These results suggested that a slightly acidic $\mathrm{pH}(6-7)$ was beneficial to intracellular lipid accumulation, whereas a slightly alkaline $\mathrm{pH}(7-8)$ was more advantageous for fast VFA adaptation and cell proliferation. In general, the discrepancies of lipid production from $\mathrm{pH} 6$ to 9 were not obvious. However, for cultures with an initial $\mathrm{pH}$ of 5 and 4, biomass and lipid production greatly decreased, and the lag phase was prolonged to approximately 1 and 3 days, respectively. Moreover, no cell growth was observed at $\mathrm{pH} 3$. These phenomena showed that cell growth and lipid accumulation by $Y$. lipolytica was greatly inhibited under such acidic conditions $(\mathrm{pH}<5)$. Given that $Y$. lipolytica could perform well under this acidic condition ( $\mathrm{pH} 4-5)$ when other carbon sources, such as glucose and glycerol, were used, the inhibitory effect of the unionized form of acetic acid, which prevailed in the acidic environment, should be the most detrimental to growth rather than the unfavorable $\mathrm{pH}$ itself. Besides these acidic $\mathrm{pH}$ conditions, an 


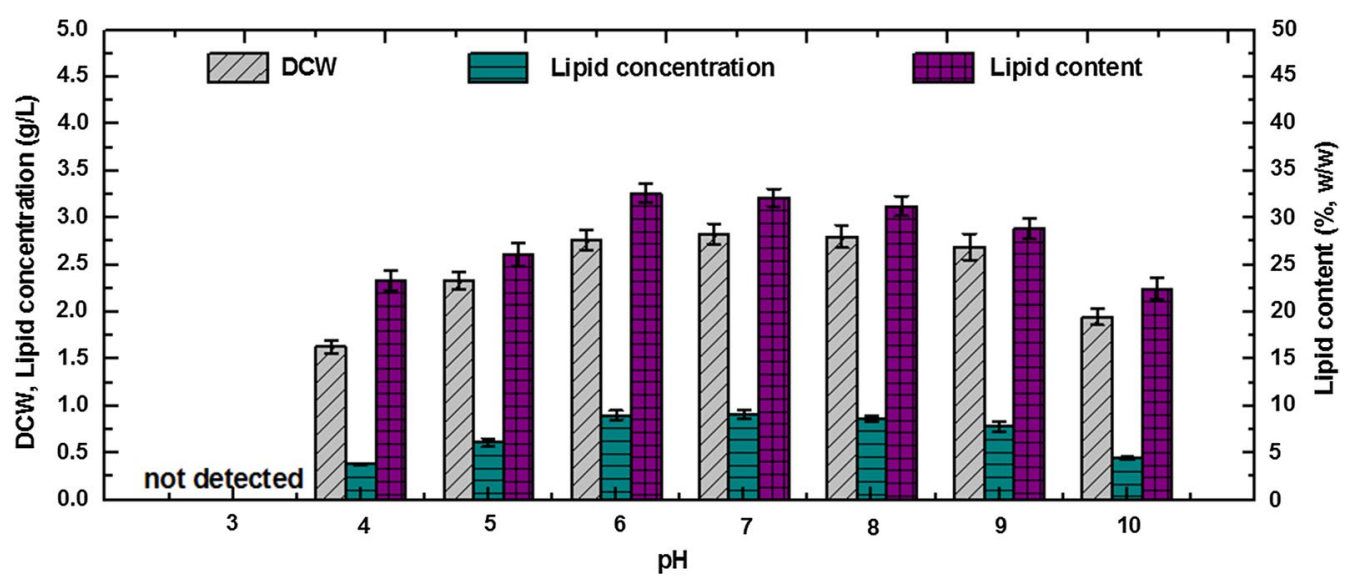

Fig. 1 The effect of initial pH on biomass and lipid production by Y. lipolytica with $3 \mathrm{~g} / \mathrm{L}$ of acetic acid as carbon source. DCW dry cell weight

initial $\mathrm{pH}$ of 10 failed to support good biomass and lipid production, and the lag phase was prolonged to 2-3 days. This result indicated that an excessively alkaline $\mathrm{pH}$ $(\geq 10)$ could also be unfavorable to $Y$. lipolytica. VFA consumption was tested after biomass was harvested. Although the specific consumption rate, $Y_{\mathrm{X} / \mathrm{S}}$, and $Y_{\mathrm{L} / \mathrm{S}}$ were different, acetic acid was consumed completely by oleaginous cells in all the the cultures that achieved cell growth within a broad $\mathrm{pH}$ range of 4-10. Fei et al. [4] showed that $2 \mathrm{~g} / \mathrm{L}$ of VFAs is consumed completely by C. albidus at neutral $\mathrm{pH}$, whereas only $<50 \%$ of VFAs is assimilated at acidic or alkaline $\mathrm{pH}$. The discrepancy may be due to the different strains used for lipid accumulation and reflects the advantage of $Y$. lipolytica.

These results suggested that $Y$. lipolytica could adapt to a wide environment $\mathrm{pH}(4-10)$ when low-concentration acetic acid was used as sole carbon source. Cultures with an initial pH from 6 to 9 had good and comparable abilities in cell growth and lipid accumulation. The best lipid production performance occurred at neutral or slightly acidic $\mathrm{pH}(6-7)$. These results further indicated that the inhibitory effects of the undissociated acid under a slightly acidic $\mathrm{pH}(6-7)$ were quite weak and negligible because of the low concentrations of VFAs and the adaptation and regulation capacity of strains. Thus, neutral and slightly acidic conditions were most commonly adopted in previous studies and recommended by authors when low-concentration (2-10 g/L) VFAs were used as a carbon source.

\section{Effects of alkaline conditions on the growth and lipid production of $Y$. lipolytica using high-content acetic acid as a carbon source}

High concentrations of VFAs cannot be efficiently converted into lipids by oleaginous yeasts $[3,17]$. The lethal effect of the high concentrations of VFAs on yeast cells has become a notable bottleneck that prevents high lipid yields and their large-scale applications; hence, further research is needed to solve this problem [14]. Acetic acid is predominantly present in waste-derived VFA mixtures [18] and thus was chosen as a model substrate in this section.

Although the adverse effects of a low VFA concentration could be weak and negligible at $\mathrm{pH} 6.0$ (a classical value used in literature), the toxicity of high VFA concentrations should be considered. Moreover, even a slightly acidic $\mathrm{pH}$ environment could further aggravated this inhibition as VFAs predominate in an unionized form in an acidic medium. An alkaline environment could be assumed to effectively alleviate the severe effect of highcontent VFAs by significantly increasing the dissociation degree of VFAs in the culture media. The cell growth and lipid accumulation of $Y$. lipolytica, five initial concentrations of acetic acid $(30,50,70,90$, and $110 \mathrm{~g} / \mathrm{L})$, and five levels of initial $\mathrm{pH}(6,7,8,9$, and 10) were tested through batch cultures at $28{ }^{\circ} \mathrm{C}$ and $180 \mathrm{rpm}$ to verify this assumption and further study the combined effects of high VFA concentrations and different alkaline conditions on VFA utilization. The results are summarized in Table 1 and analyses are as below.

\section{Level of acid that $Y$. lipolytica could tolerate and consume}

As demonstrated in Table 1, the $\mathrm{pH}$ of the cultivation environment had crucial effects on $Y$. lipolytica when high-content acetic acid was used as a carbon source. At an initial $\mathrm{pH}$ of 6 , only cultures with $30 \mathrm{~g} / \mathrm{L}$ acetic acid achieved cell growth and lipid accumulation. No cell growth was observed in cultures with other concentrations (50, 70, 90 and $110 \mathrm{~g} / \mathrm{L})$ of acetic acid at $\mathrm{pH} 6$, which reflects that the toxicity of such high 
Table 1 Biomass and lipid production of $Y$. lipolytica with high concentrations of acetic acid as carbon source at different pH conditions

\begin{tabular}{|c|c|c|c|c|c|c|}
\hline $\begin{array}{l}\text { Carbon source } \\
\text { and initial pH }\end{array}$ & Biomass (g/L) & Lipid conc. (g/L) & Lipid content (wt\%) & $Y_{\mathrm{x} / \mathrm{s}}(\mathrm{g} / \mathrm{g})$ & $Y_{\mathrm{L} / \mathrm{S}}(\mathrm{g} / \mathrm{g})$ & Lag phase (day) \\
\hline \multicolumn{7}{|c|}{$30 \mathrm{~g} / \mathrm{L}$ of acetic acid } \\
\hline $\mathrm{pH}=6$ & $10.56 \pm 0.73$ & $1.73 \pm 0.08$ & $16.41 \pm 1.08$ & $0.352 \pm 0.024$ & $0.058 \pm 0.003$ & $3-5$ \\
\hline $\mathrm{pH}=7$ & $17.28 \pm 0.85$ & $5.16 \pm 0.19$ & $29.89 \pm 0.89$ & $0.576 \pm 0.028$ & $0.172 \pm 0.006$ & $0^{\mathrm{a}}$ \\
\hline $\mathrm{pH}=8$ & $17.86 \pm 0.76$ & $5.24 \pm 0.23$ & $29.33 \pm 0.86$ & $0.595 \pm 0.025$ & $0.175 \pm 0.008$ & 0 \\
\hline $\mathrm{pH}=9$ & $17.05 \pm 0.61$ & $4.57 \pm 0.25$ & $26.81 \pm 1.07$ & $0.568 \pm 0.020$ & $0.152 \pm 0.008$ & 0 \\
\hline $\mathrm{pH}=10$ & $13.67 \pm 0.58$ & $2.75 \pm 0.11$ & $20.14 \pm 1.46$ & $0.456 \pm 0.019$ & $0.092 \pm 0.004$ & 0 \\
\hline \multicolumn{7}{|c|}{$50 \mathrm{~g} / \mathrm{L}$ of acetic acid } \\
\hline $\mathrm{pH}=6$ & N.D. & N.D. & N.D. & N.D. & N.D. & N.D. \\
\hline $\mathrm{pH}=7$ & $27.02 \pm 1.21$ & $7.40 \pm 0.25$ & $27.38 \pm 0.83$ & $0.540 \pm 0.024$ & $0.148 \pm 0.005$ & $0-1$ \\
\hline $\mathrm{pH}=8$ & $28.92 \pm 1.15$ & $8.01 \pm 0.30$ & $27.69 \pm 0.96$ & $0.578 \pm 0.023$ & $0.160 \pm 0.006$ & 0 \\
\hline $\mathrm{pH}=9$ & $27.39 \pm 1.22$ & $7.10 \pm 0.28$ & $25.93 \pm 1.35$ & $0.548 \pm 0.024$ & $0.142 \pm 0.006$ & $0-1$ \\
\hline $\mathrm{pH}=10$ & $17.07 \pm 1.09$ & $3.23 \pm 0.09$ & $18.95 \pm 0.94$ & $0.341 \pm 0.022$ & $0.065 \pm 0.002$ & $1-3$ \\
\hline \multicolumn{7}{|c|}{$70 \mathrm{~g} / \mathrm{L}$ of acetic acid } \\
\hline $\mathrm{pH}=6$ & N.D. & N.D. & N.D. & N.D. & N.D. & N.D. \\
\hline $\mathrm{pH}=7$ & $29.10 \pm 1.45$ & $6.76 \pm 0.36$ & $23.23 \pm 1.04$ & $0.497 \pm 0.025$ & $0.115 \pm 0.006$ & $1-3$ \\
\hline $\mathrm{pH}=8$ & $37.14 \pm 1.56$ & $10.11 \pm 0.42$ & $27.22 \pm 0.92$ & $0.531 \pm 0.022$ & $0.144 \pm 0.006$ & 0 \\
\hline $\mathrm{pH}=9$ & $35.67 \pm 1.64$ & $9.09 \pm 0.40$ & $25.49 \pm 1.11$ & $0.510 \pm 0.023$ & $0.130 \pm 0.006$ & $2-3$ \\
\hline $\mathrm{pH}=10$ & $13.35 \pm 0.83$ & $1.84 \pm 0.13$ & $13.75 \pm 1.50$ & $0.269 \pm 0.017$ & $0.037 \pm 0.003$ & $3-5$ \\
\hline \multicolumn{7}{|c|}{$90 \mathrm{~g} / \mathrm{L}$ of acetic acid } \\
\hline $\mathrm{pH}=6$ & N.D. & N.D. & N.D. & N.D. & N.D. & N.D. \\
\hline $\mathrm{pH}=7$ & $19.96 \pm 0.88$ & $4.36 \pm 0.18$ & $21.85 \pm 1.01$ & $0.291 \pm 0.013$ & $0.064 \pm 0.003$ & $1-3$ \\
\hline $\mathrm{pH}=8$ & $31.02 \pm 1.22$ & $7.90 \pm 0.37$ & $25.47 \pm 1.23$ & $0.417 \pm 0.016$ & $0.106 \pm 0.005$ & $0-1$ \\
\hline $\mathrm{pH}=9$ & $24.19 \pm 1.19$ & $5.92 \pm 0.32$ & $24.46 \pm 1.40$ & $0.319 \pm 0.016$ & $0.078 \pm 0.004$ & $3-4$ \\
\hline $\mathrm{pH}=10$ & $9.606 \pm 0.86$ & $1.38 \pm 0.06$ & $14.32 \pm 1.07$ & $0.171 \pm 0.015$ & $0.024 \pm 0.001$ & $6-7$ \\
\hline \multicolumn{7}{|c|}{$110 \mathrm{~g} / \mathrm{L}$ of acetic acid } \\
\hline $\mathrm{pH}=6$ & N.D. & N.D. & N.D. & N.D. & N.D. & N.D. \\
\hline $\mathrm{pH}=7$ & $15.80 \pm 0.93$ & $2.49 \pm 015$ & $15.75 \pm 0.89$ & $0.192 \pm 0.011$ & $0.030 \pm 0.002$ & $4-5$ \\
\hline $\mathrm{pH}=8$ & $17.33 \pm 0.62$ & $3.85 \pm 0.19$ & $22.21 \pm 1.28$ & $0.198 \pm 0.007$ & $0.044 \pm 0.002$ & 2 \\
\hline $\mathrm{pH}=9$ & $15.40 \pm 0.77$ & $3.30 \pm 0.19$ & $21.45 \pm 1.53$ & $0.177 \pm 0.009$ & $0.038 \pm 0.002$ & $3-5$ \\
\hline $\mathrm{pH}=10$ & $10.20 \pm 1.23$ & $1.19 \pm 0.10$ & $11.67 \pm 1.03$ & $0.167 \pm 0.020$ & $0.019 \pm 0.002$ & $9-10$ \\
\hline
\end{tabular}

All the results presented are the mean values $\pm S D$ for three independent replicates

N.D., not detected; $Y_{\mathrm{X} / \mathrm{s}}$, growth yield coefficient, g DCW/g VFAs, $Y_{\mathrm{L} / \mathrm{s}}$, lipid yield coefficient, $\mathrm{g}$ lipid/g VFAs

${ }^{a}$ Lag phase less than $3 \mathrm{~h}$ was recorded as 0 day

concentrations of acetic acid under this acidic environment is intolerable to $Y$. lipolytica. However, all the cultures at an initial $\mathrm{pH}$ of $7-10$ and at $30-110 \mathrm{~g} / \mathrm{L}$ acetic acid concentrations achieved growth resumption and lipid production. These results demonstrated that increasing the medium $\mathrm{pH}$ to alkaline levels greatly alleviated the toxicity of high-content acetic acid and thus greatly increased the acidic level that $Y$. lipolytica could tolerate.

Acetic acid consumption by Y. lipolytica in all the cultures was tested after the biomass was harvested. The corresponding VFA utilization ratios (\%) were calculated (Fig. 2c). When 30 and $50 \mathrm{~g} / \mathrm{L}$ of acetic acid was used as the sole carbon source, all the cultures that achieved biomass and lipid production consumed acid completely. As the acetic acid concentration increased, the utilization ratios had varying degrees of decline even though acid consumption $(\mathrm{g} / \mathrm{L})$ increased accordingly. When $70 \mathrm{~g} / \mathrm{L}$ of acetic acid was used as a carbon source, only cultures at $\mathrm{pH} 8$ and 9 completely consumed acetic acid, whereas the utilization ratio (\%) at $\mathrm{pH} 7$ and 10 declined to $83.73 \%$ and $79.84 \%$, respectively. For cultures with 90 and $110 \mathrm{~g} / \mathrm{L}$ acetic acid, $Y$. lipolytica could not completely assimilate acetic acid at all $\mathrm{pH}$ levels, whereas the highest consumption and utilization ratios were obtained at $\mathrm{pH} 8-9$. 

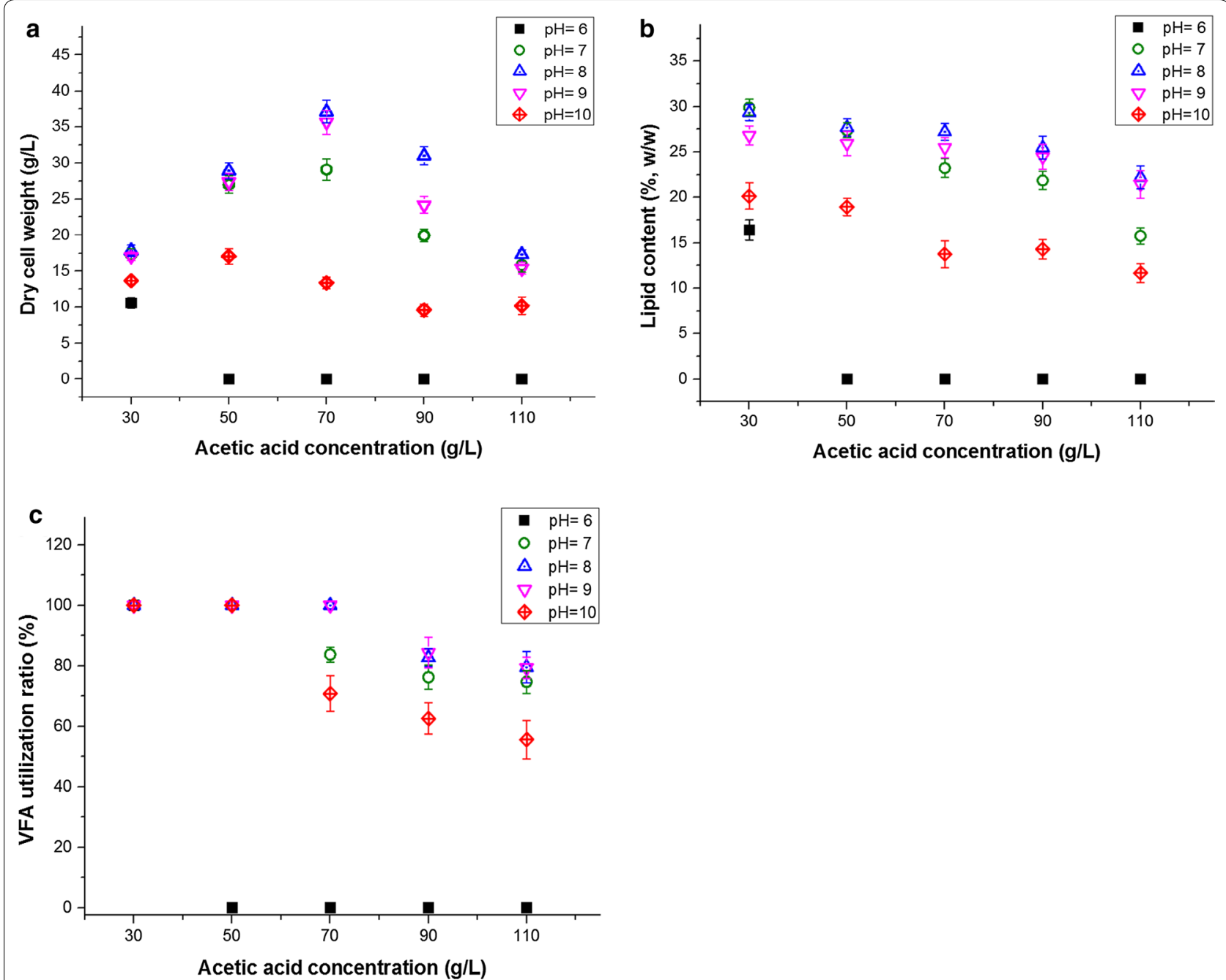

Fig. 2 Comparison of a dry cell weight, $\mathbf{b}$ lipid content, and $\mathbf{c} V F A$ utilization ratio obtained during batch cultivation with different high concentrations of acetic acid as carbon source under different initial pH conditions

It is worth noting that as the acetic acid concentration increased, the optimal medium $\mathrm{pH}$ for the growth of $Y$. lipolytica increased to a certain extent. For cultures with 30 and $50 \mathrm{~g} / \mathrm{L}$ acetic acid, a medium $\mathrm{pH}$ of 7-8 was recommended on the basis of biomass and lipid production, whereas cultures at $\mathrm{pH}$ 8-9 had improved performances when the concentration of acetic acid was $\geq 70 \mathrm{~g} / \mathrm{L}$. However, these results did not indicate that the higher the concentrations of VFA were, the more alkaline the condition would be. From pH 7 to 10 , cultures with all these high concentrations of acetic acid as the carbon source had the worst performance at $\mathrm{pH} 10$. This phenomenon was explained by abovementioned conclusions, which elucidated that $\mathrm{pH}$ 10 adversely affected the cell growth and lipid accumulation of Y. lipolytica.

\section{Lag phase and growth curve}

Short lag phases were observed in an alkaline environment when high concentrations of acetic acid were used as a carbon source (Table 1). For cultures with $30 \mathrm{~g} / \mathrm{L}$ acetic acid, approximately $3-5$ days of adaptation was needed for the yeast to resume growth at $\mathrm{pH} 6$. However, at $\mathrm{pH}$ 7-10, Y. lipolytica cells started proliferating without an obvious lag phase $(<3 \mathrm{~h})$, indicating that the adverse effect of acetic acid on cell proliferation was basically eliminated. As the acetic acid concentration increased, the lag phase under different $\mathrm{pH}$ conditions was extended to varying degrees. It is found that cultures at $\mathrm{pH} 8$ always had the shortest lag phase. Specifically, at an initial $\mathrm{pH}$ of 8 , no evident lag phase was observed at $\leq 70 \mathrm{~g} / \mathrm{L}$ acetic acid. The longest lag phase, which was observed at $110 \mathrm{~g} / \mathrm{L}$ acetic acid, was achieved only in approximately 2 days. Based 
on $\mathrm{pH} 8$, the increase and decrease in $\mathrm{pH}$ could prolong the period of lag phase. At the same acetic acid concentration, the cultures at $\mathrm{pH} 10$ had the longest lag phase compared with those at $\mathrm{pH} 7$ and 9.

Microorganisms can respond and adapt to harsh environments via a series of adaptive mechanisms. However, this capacity for adaptation and regulation is limited [17]. High acetic acid concentrations can exhibit adverse and even lethal effect on yeast cell growth. Our results showed that an alkaline medium $\mathrm{pH}$ could effectively alleviate the growth inhibition and increase the tolerance level of Y. lipolytica to high-content acetic acid. Nevertheless, an alkaline environment could not completely eliminate the severe effect of a high acid concentration ( $>70 \mathrm{~g} / \mathrm{L})$. Moreover, an excessive alkaline environment $(\mathrm{pH} \geq 10)$ negatively affected yeast growth. OD 600 (data not shown) in all the cultures was measured throughout the cultivation duration. Interestingly, the growth curve of $Y$. lipolytica with the imposed non-negligible acid stress under acidic and alkaline conditions exhibited different features. When suffering under severe but tolerable inhibitory effects by acetic acid, Y. lipolytica cultured in an alkaline medium could achieve growth resumption after a short-term lag phase, whereas the slope of the growth curve in the exponential phase was usually not large. On the contrary, Y. lipolytica cultured in an acidic medium needed a much longer period of adaptation to resist growth inhibition if the inhibitory effect was tolerable. However, when growth was resumed, the slope of the growth curve in the exponential phase was larger than that in the cultures in an alkaline medium. These discrepancies could be explained by the different inhibitory mechanisms of acid and yeast responses under different $\mathrm{pH}$ conditions. The inhibitory effects of VFAs under alkaline conditions, which mainly stem from anion (dissociated weak acid) accumulation or excessive alkaline $\mathrm{pH}$, were usually not lethal. The strain could easily resist damage, but the cell proliferation rate was likely impaired because of low vitality. However, in the case of cultures under acidic conditions, the strain experienced difficulty in surviving because of inhibitory effects due to cytosolic acidification by uncoupling could be fatal. Under this extreme circumstance, the adaptation period is markedly prolonged and usually accompanied by changes in membrane structure and gene mutations [30-33]. Once adaptation was successful, the growth resumption would proceed at a fast cell proliferation rate.

\section{Biomass and lipid production}

The effects of different initial $\mathrm{pH}$ values on the biomass production of cultures with high-content acetic acid as a carbon source are illustrated in Fig. 2a. In accordance with the results in "Level of acid that $Y$. lipolytica could tolerate and consume" and "Lag phase and growth curve" sections, $Y$. lipolytica could accumulate more biomass under alkaline conditions of $\mathrm{pH} \mathrm{7-9}$ and obtain the highest biomass at $\mathrm{pH}$ 8. Acetic acid concentration considerably affected biomass production. With concentrations $<70 \mathrm{~g} / \mathrm{L}$, the increase in acetic acid concentration caused an increase in the biomass, whereas cultures with $>70 \mathrm{~g} / \mathrm{L}$ acetic acid had a decreased biomass. The highest biomass production $(37.14 \mathrm{~g} / \mathrm{L})$ was obtained with $70 \mathrm{~g} / \mathrm{L}$ acetic acid at an initial $\mathrm{pH}$ of 8 .

These results indicated that the alkaline condition for alleviating the inhibitory effects of high-concentration VFAs had a certain concentration limit. This limitation was related to the mechanism of the remission effect of an alkaline environment. This mechanism involved alleviating the inhibitory effect attributed to uncoupling by significantly increasing the dissociation degree of weak acids. However, this mechanism could not work on anion accumulation. It is generally accepted that the lethal effects of weak acids occur due to the lowering of microorganism's internal $\mathrm{pH}$ by uncoupling, while some researchers argue that the intracellular accumulation of the dissociated weak acid (anion) itself plays the most significant role in cell death [32]. The results in the present study indicated that uncoupling plays a major role in the lethal effect on Y. lipolytica cultured with VFAs, whereas anion accumulation could have an adverse effect only when its concentration was high.

As shown in Fig. 2b, for cultures at all the pH conditions, the highest lipid content was obtained with $30 \mathrm{~g} / \mathrm{L}$, from $\mathrm{pH} 6$ to 10, which was 16.41, 29.89, 29.33, 26.81 and $20.14 \%$, respectively. In contrast to the changes observed in biomass, the lipid content showed a trend of unilateral decline as the acetic acid concentration increased. This trend was consistent with our previous study [17], which involved culturing $Y$. lipolytica with low concentrations $(<20 \mathrm{~g} / \mathrm{L})$ of VFA under a slightly acidic $\mathrm{pH}$ of 6. However, under alkaline conditions in this study, the downward trend of the lipid content was much gentle. In particular, at $\mathrm{pH} 8$ and 9, the cultures with $110 \mathrm{~g} / \mathrm{L}$ acetic acid could maintain $>20 \%$ of their lipid content. On the contrary, under slightly acidic and neutral conditions of pH 6 and 7, Y. lipolytica lipid accumulation was clearly inhibited as the acid concentration increased.

In general, lipid production depends on both biomass and lipid content. When the acid concentration increased from 30 to $110 \mathrm{~g} / \mathrm{L}$, the obtained lipid concentration initially increased and then decreased (Table 1 ). The highest lipid production $(10.11 \mathrm{~g} / \mathrm{L})$ was obtained with $70 \mathrm{~g} / \mathrm{L}$ acetic acid at an initial $\mathrm{pH}$ of 8 . Notably, besides the increased biomass and lipid production, the growth yield coefficient $\left(Y_{\mathrm{X} / \mathrm{S}}\right)$ and lipid yield coefficient $\left(Y_{\mathrm{L} / \mathrm{S}}\right)$ of $Y$. lipolytica cultured with such high 
acetic acid concentrations were greatly improved under the proper alkaline conditions determined in this study. With $\leq 70 \mathrm{~g} / \mathrm{L}$ acetic acid, $Y_{\mathrm{X} / \mathrm{S}}$ could exceed 0.50 , and $Y_{\mathrm{L} / \mathrm{S}}$ could exceed 0.11 by adjusting the medium $\mathrm{pH}$ to 7-9. However, in our previous study [17], at $\mathrm{pH} 6$, cultures with $20 \mathrm{~g} / \mathrm{L}$ acetic acid could only achieve $Y_{\mathrm{X} / \mathrm{S}}$ of 0.37 and $Y_{\mathrm{L} / \mathrm{S}}$ of 0.05 . The significant improvement of $Y_{\mathrm{X} / \mathrm{S}}$ and $Y_{\mathrm{L} / \mathrm{S}}$ further elucidated that the increased biomass and lipid yield was not only because of the abundant carbon source, but more importantly, due to the improved viability and vitality of $Y$. lipolytica under alkaline conditions against the imposed acid stress.

\section{Change in pH during cultivation}

The nutrient consumption and metabolism of yeast can change the $\mathrm{pH}$ in culture media. The $\mathrm{pH}$ of all the cultures was determined every day after inoculation. It is found that the $\mathrm{pH}$ of the cultures that did not have cell growth remained almost unchanged during the cultivation period. The cultures showing biomass and lipid production demonstrated similar trends in $\mathrm{pH}$ change. Figure 3a shows the changes in the $\mathrm{pH}$ of the cultures with $50 \mathrm{~g} / \mathrm{L}$ acetic acid at different initial $\mathrm{pH}$ values. As depicted, the $\mathrm{pH}$ of the cultures at an initial $\mathrm{pH}$ of 6 remained almost unchanged. The $\mathrm{pH}$ in all of the cultures at an initial $\mathrm{pH}$ of 7-10 had varying degrees of decline to reach the lowest point on the first few days, and then gradually increased, and finally stabilized at $\mathrm{pH}$ 9.1-9.4. Lian et al. [6] and Zheng et al. [28] indicated that the massive uptake of ammonium leads to a rapid $\mathrm{pH}$ decrease on the first few days, whereas the consumption of acetate ions $\left(\mathrm{CH}_{3} \mathrm{COO}^{-}\right)$and the production of $\mathrm{OH}^{-}$ groups result in a $\mathrm{pH}$ increase on the following days.

In the current study, the lowest $\mathrm{pH}$ of the cultures varied with different initial $\mathrm{pH}$ values. Notably, for cultures at an initial $\mathrm{pH}$ of 7 , the lowest $\mathrm{pH}$ point could nearly

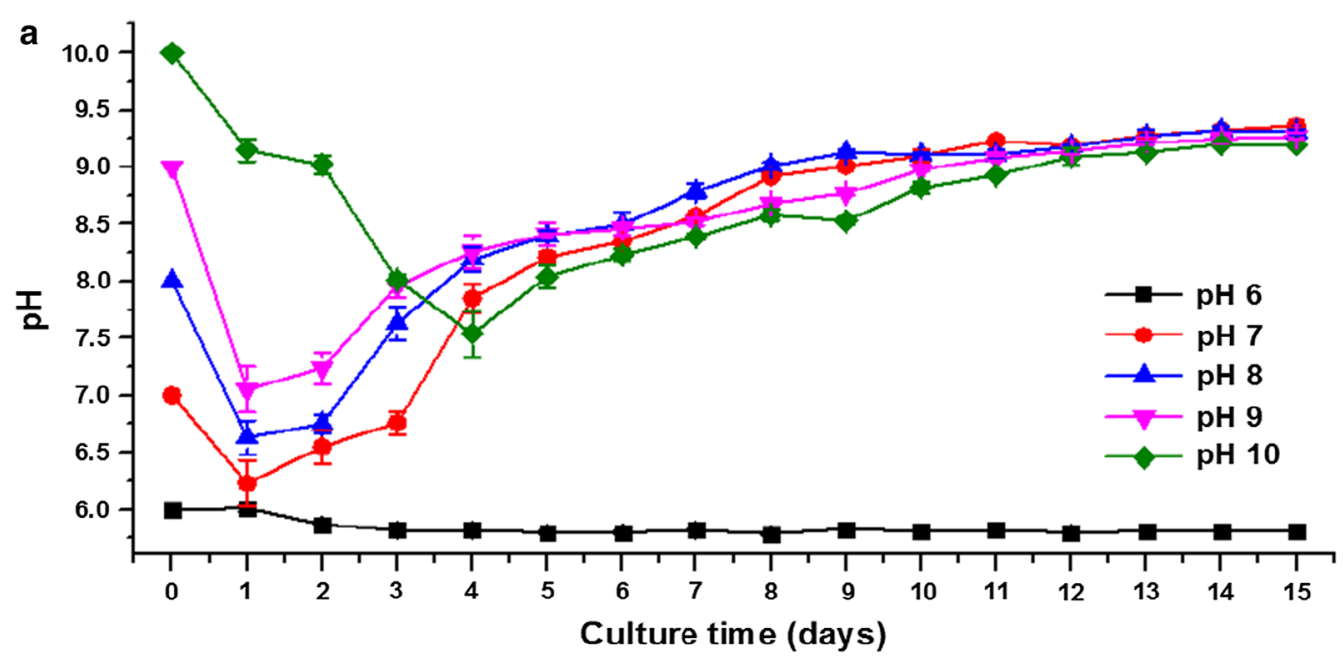

b

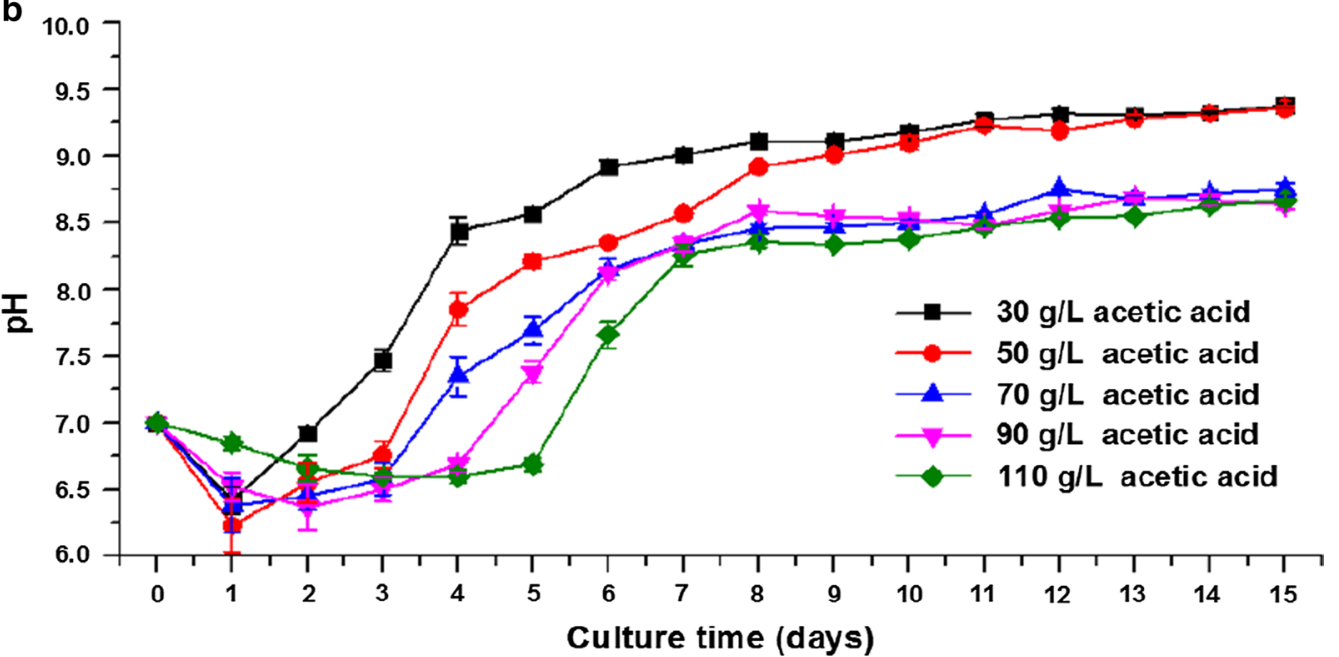

Fig. 3 The change of $\mathrm{pH}$ in cultures a with $50 \mathrm{~g} / \mathrm{L}$ of acetic acid at different initial pH, $\mathbf{b}$ with $30-110 \mathrm{~g} / \mathrm{L}$ of acetic acid at an initial pH of 7 
drop to $6(\mathrm{pH} 6.23 \pm 0.20)$. Acetic acid, propionic acid, and butyric acid have pKa of 4.76, 4.87, and 4.82, respectively, indicating that $99 \%$ of these VFAs appear in the dissociated form at $\mathrm{pH}$ 7. However, such a decrease in $\mathrm{pH}$ to an acidic level at the early stage of cultures could largely increase the amount of undissociated VFAs in the system and their inhibitory effects. This inhibition could adversely affect yeast cell proliferation and lipid accumulation, causing the system $\mathrm{pH}$ to change slowly, prolonging the lag phase, and changing lipid production performance. Figure $3 \mathrm{~b}$ illustrates the changes in the $\mathrm{pH}$ of the cultures with $30-110 \mathrm{~g} / \mathrm{L}$ acetic acid at an initial $\mathrm{pH}$ of 7 . This inhibition and its adverse effect were more obvious in cultures with a higher acetic acid concentration. This occurrence could also explain that the optimal initial $\mathrm{pH}$ was more alkaline to a certain extent to avoid the inhibitory effect from the undissociated acid caused by a drop in medium $\mathrm{pH}$ when the acetic acid concentration was too high $(>70 \mathrm{~g} / \mathrm{L})$.

As shown in Fig. 3a, irrespective of the initial $\mathrm{pH}$ or changes in $\mathrm{pH}$ during the cultivation period, the final fermentation $\mathrm{pH}$ of cultures which realize biomass and lipid accumulation reached at an equivalent level. This result was inconsistent with studies by Santamauro et al. [34] and Zheng et al. [28], who obtained different final $\mathrm{pH}$ values by using other types of organic waste to cultivate $M$. pulcherrima and C. sorokiniana at different initial $\mathrm{pH}$ values, respectively. This discrepancy may be due to the different strains and feedstock used, and further metabolic level studies are needed to elucidate the specific mechanisms of $Y$. lipolytica on regulating intracellular and extracellular $\mathrm{pH}$ when VFAs are used as carbon sources. Lian et al. [6] revealed that the initial acetate concentration unlikely affects the final fermentation $\mathrm{pH}$ when fermentation is carried out with C. curvatus. In the present work, as demonstrated in Fig. 3b, the initial VFA concentration did not affect the final $\mathrm{pH}$ in the cultures that completely consumed VFAs (cultures with 30 and $50 \mathrm{~g} / \mathrm{L}$ acetic acid). The cultures with incomplete VFA consumption resulted in a relatively low final fermentation $\mathrm{pH}$.

\section{Growth and lipid production of $Y$. lipolytica using high-content propionic acid, butyric acid, and mixed VFAs as carbon sources under an alkaline condition}

The concentration of waste-derived VFAs is generally no more than $50 \mathrm{~g} / \mathrm{L}$, and the composition ratio of VFAs can be affected by substrate types and fermentation conditions, with acetic acid, propionic acid, and butyric acid as the main components [8-13]. Batch cultures were conducted by using $50 \mathrm{~g} / \mathrm{L}$ propionic acid, butyric acid, and a synthetic acetic acid/propionic acid/butyric acid (5:2:3) VFA mixture as carbon sources with an initial $\mathrm{pH}$ of 8 to evaluate the feasibilities of the two other typical VFA types and a mixed system. The results are illustrated in Figs. 4 and 5.

The growth curve (Fig. 4a) showed that all of the batches started cell proliferation without an evident lag phase, indicating that the growth inhibitory effects of these high-content VFAs on Y. lipolytica were effectively relieved under this alkaline condition. The cell growth rates on acetic acid and mixed acid were slightly greater than those on propionic acid and butyric acid during the exponential phase. The differences in cell growth rates were directly associated with the different utilization rates of VFAs (Fig. 4b), which were attributed to various metabolic pathways of different single VFA after intake. Acetic acid can be directly transformed to acetyl-CoA, a central intermediate in lipid synthesis [20]. Propionate, an odd-chain carboxylic acid, should be converted to propionyl-CoA and then enter the tricarboxylic acid cycle via the interconversion of methylmalonyl-CoA to succinyl-CoA [7]. Butyrate must undergo a number of biochemical transformations, including $\beta$-oxidation to acetoacetyl-CoA, and further cleave to acetyl-CoA [35]. In our study, the biomass and lipids produced were harvested at the end of the stationary phase. As demonstrated in Fig. 4c, the cultures on acetic acid, butyric acid, and mixed acids had comparable biomass and lipid production, whereas the cultures on propionic acid showed a much inferior performance, especially biomass production. The concentrations of the biomass produced in the cultures with acetic acid, butyric acid, and mixed acid were 28.92, 28.52, $27.33 \mathrm{~g} / \mathrm{L}$, respectively. By comparison, the concentration of the biomass produced in the cultures with propionic acid was only $18.11 \mathrm{~g} / \mathrm{L}$. The lipid contents slightly differed. The highest lipid content was observed in mixed acid (30.25\%), followed by acetic acid (27.69\%), butyric acid (25.32\%), and propionic acid (24.73\%). Thus, the cultures with $50 \mathrm{~g} / \mathrm{L}$ acetic acid, propionic acid, butyric acid, and mixed acids at an initial $\mathrm{pH}$ of 8 achieved 8.01, 4, 48, 7.22, and $8.27 \mathrm{~g} / \mathrm{L}$ lipid yields, respectively.

Yarrowia lipolytica prefers acetic acid to propionic acid and butyric acid for cell growth and lipid accumulation [17]. In our previous research, 2.5-20 g/L VFAs were used as carbon sources in a slightly acidic environment $(\mathrm{pH}=6)$, and our results revealed that the inhibitory effects of the same concentrations of propionic acid and butyric acid were much more severe than those of acetic acid. Thus, Y. lipolytica could only tolerate low concentrations of propionic acid $(\leq 10 \mathrm{~g} / \mathrm{L})$ and butyric acid $(\leq 5 \mathrm{~g} / \mathrm{L})$ with low biomass and lipid production. However, an alkaline medium $(\mathrm{pH}=8)$ was used in this study, so the levels of propionic acid and butyric acid that $Y$. lipolytica could tolerate and the corresponding biomass 

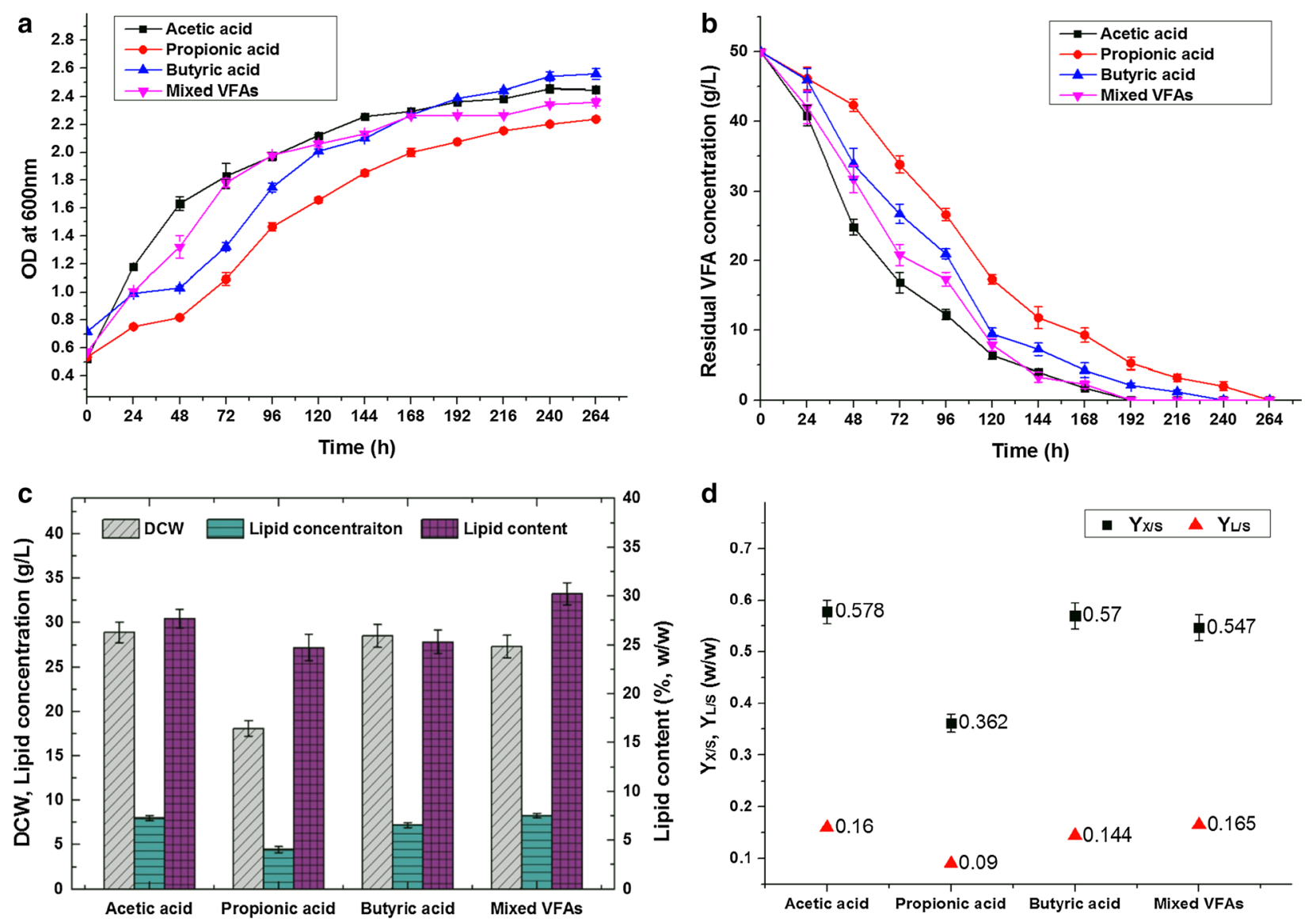

Fig. 4 Profiles of a growth curve, $\mathbf{b} V F A$ consumption, $\mathbf{c}$ biomass and lipid production, and $\mathbf{d}$ growth yield coefficient $\left(Y_{X / S}\right)$ and lipid yield coefficient $\left(Y_{\mathrm{L} / \mathrm{S}}\right)$ obtained during batch cultivation with $50 \mathrm{~g} / \mathrm{L}$ of different types of VFA as carbon source at an initial pH of 8.0

and lipid production remarkably improved. Interestingly, $Y_{\mathrm{X} / \mathrm{S}}(0.570)$ and $Y_{\mathrm{L} / \mathrm{S}}(0.144)$ of $Y$. lipolytica using butyric acid as its carbon source reached a comparable level with those of $Y$. lipolytica utilizing acetic acid as its carbon source $(0.578,0.160)$. Thus, comparable biomass and lipid yields were obtained. According to our supplementary experimental results at other $\mathrm{pH}$ levels $(\mathrm{pH} 6-10$, Table 2), the performance of the cultures at $\mathrm{pH} 8-10$ was better than that of the cultures at $\mathrm{pH} 7$ when $50 \mathrm{~g} / \mathrm{L}$ butyric acid was used as a carbon source. Conversely, the performance of the cultures at $\mathrm{pH}$ 7-9 was better than that of the cultures at $\mathrm{pH} 10$ when acetic acid and propionic acid were used as carbon sources. Notably, the cultures with butyric acid at $\mathrm{pH} 10$ could still perform well. These results indicated that a more alkaline $\mathrm{pH}$ compared with that of acetic acid and propionic acid was beneficial to the bioconversion of high-content butyric acid to lipids by $Y$. lipolytica.

A mixture of acetic acid, propionic acid, and butyric acid (5:2:3) was used as sole carbon sources with a total VFA concentration of $50 \mathrm{~g} / \mathrm{L}$ to evaluate acid utilization and lipid production by Y. lipolytica using high-content waste-derived VFAs. The VFA ratio was identified on the basis of our previously reported results focusing on the anaerobic fermentation of FW materials [17]. Although certain levels of unfavorable acids, such as propionic acid, were found, the cultures with mixed VFAs favored good growth and lipid accumulation profiles. In Fig. 4c, the cultures with the mixed VFAs had the highest lipid yield $(8.27 \mathrm{~g} / \mathrm{L})$ and the highest lipid content $(30.25 \%)$. The biomass production on mixed VFAs was $27.33 \mathrm{~g} / \mathrm{L}$. This value was slightly lower but still comparable with that in cultures with single acetic acid and butyric acid, which were 28.92 and $28.52 \mathrm{~g} / \mathrm{L}$, respectively. In addition, our supplementary experimental results (Table 2) indicated that the cultures with mixed VFAs could still resume growth after 2-3 days of adaptation and finally obtain a lipid yield of $3.16 \mathrm{~g} / \mathrm{L}$ even at an initial $\mathrm{pH}$ of 6 . No cell growth and lipid accumulation were observed in the cultures with a single acid under the same condition. These phenomena involved the diversity of carbon source and the specific VFA utilization of Y. lipolytica in a mixed 


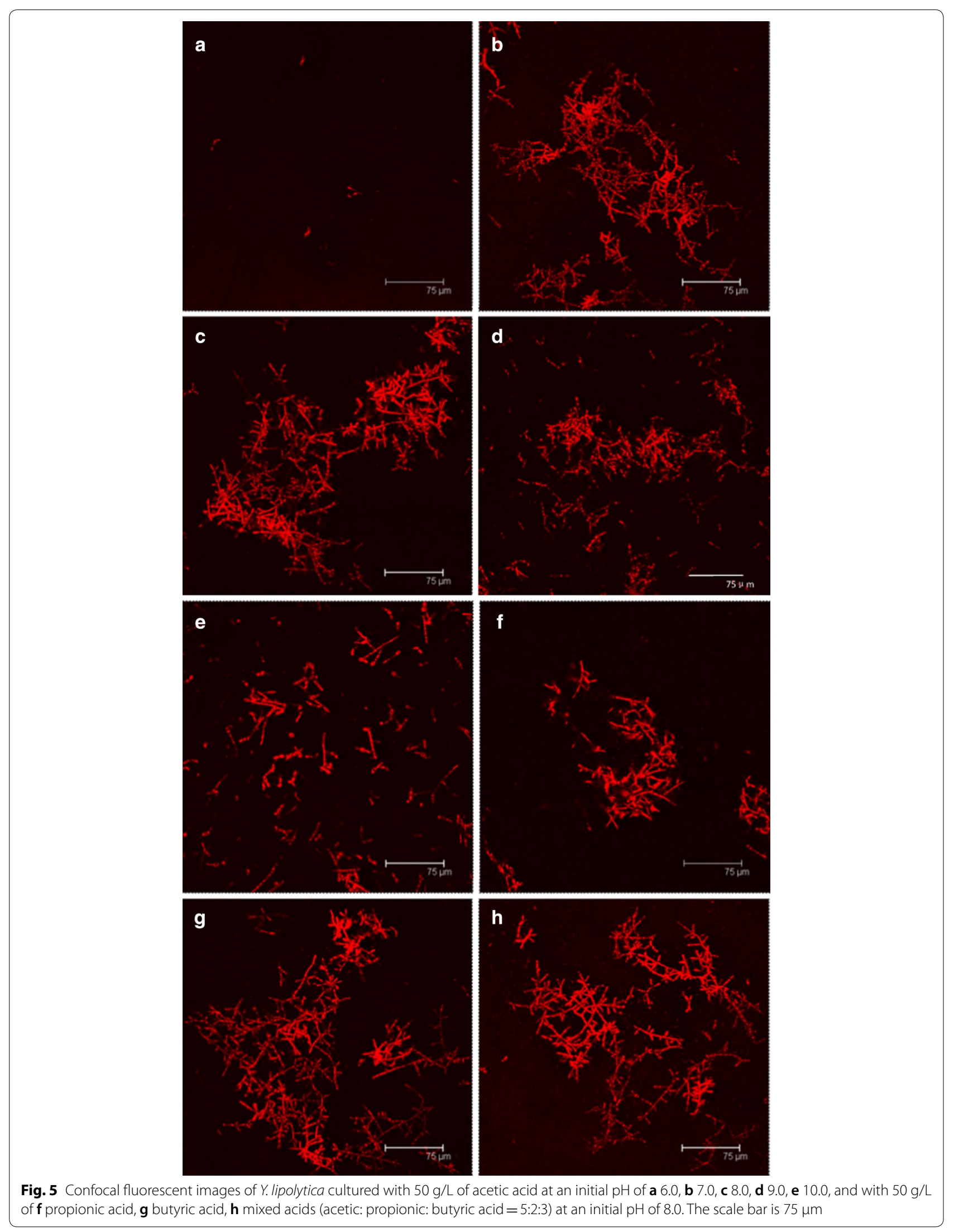


Table 2 Biomass and lipid production of $Y$. lipolytica with $50 \mathrm{~g} / \mathrm{L}$ of propionic acid, butyric acid and mixed VFAs as carbon source at different $\mathrm{pH}$ conditions

\begin{tabular}{|c|c|c|c|c|c|c|}
\hline $\begin{array}{l}\text { Carbon source } \\
\text { and initial pH }\end{array}$ & Biomass (g/L) & Lipid conc. (g/L) & Lipid content (wt\%) & $Y_{\mathrm{X} / \mathrm{s}}(\mathrm{g} / \mathrm{g})$ & $Y_{\mathrm{L} / \mathrm{S}}(\mathrm{g} / \mathrm{g})$ & Lag phase (day) \\
\hline \multicolumn{7}{|c|}{$50 \mathrm{~g} / \mathrm{L}$ of propionic acid } \\
\hline $\mathrm{pH}=6$ & N.D. & N.D. & N.D. & N.D. & N.D. & N.D. \\
\hline $\mathrm{pH}=7$ & $15.99 \pm 0.92$ & $3.83 \pm 0.21$ & $23.96 \pm 1.34$ & $0.320 \pm 0.018$ & $0.077 \pm 0.004$ & $1-2$ \\
\hline $\mathrm{pH}=8$ & $18.11 \pm 0.87$ & $4.48 \pm 0.34$ & $24.73 \pm 1.20$ & $0.362 \pm 0.017$ & $0.090 \pm 0.007$ & $0^{\mathrm{a}}$ \\
\hline $\mathrm{pH}=9$ & $17.26 \pm 1.01$ & $4.33 \pm 0.35$ & $25.06 \pm 1.47$ & $0.345 \pm 0.020$ & $0.087 \pm 0.007$ & $0-1$ \\
\hline $\mathrm{pH}=10$ & $9.67 \pm 0.69$ & $1.58 \pm 0.16$ & $16.33 \pm 1.33$ & $0.193 \pm 0.014$ & $0.032 \pm 0.003$ & $2-3$ \\
\hline \multicolumn{7}{|c|}{$50 \mathrm{~g} / \mathrm{L}$ of butyric acid } \\
\hline $\mathrm{pH}=6$ & N.D. & N.D. & N.D. & N.D. & N.D. & N.D. \\
\hline $\mathrm{pH}=7$ & $25.45 \pm 1.10$ & $6.53 \pm 0.24$ & $25.65 \pm 1.17$ & $0.509 \pm 0.022$ & $0.131 \pm 0.005$ & $1-3$ \\
\hline $\mathrm{pH}=8$ & $28.52 \pm 1.28$ & $7.22 \pm 0.27$ & $25.32 \pm 1.20$ & $0.570 \pm 0.026$ & $0.144 \pm 0.005$ & 0 \\
\hline $\mathrm{pH}=9$ & $28.92 \pm 1.28$ & $7.92 \pm 0.23$ & $27.39 \pm 1.47$ & $0.578 \pm 0.026$ & $0.158 \pm 0.005$ & 0 \\
\hline $\mathrm{pH}=10$ & $26.86 \pm 1.43$ & $7.16 \pm 0.30$ & $26.67 \pm 1.33$ & $0.537 \pm 0.029$ & $0.143 \pm 0.006$ & $1-2$ \\
\hline \multicolumn{7}{|c|}{$50 \mathrm{~g} / \mathrm{L}$ of mixed VFAs (acetic: propionic: butyric acid = 5:2:3) } \\
\hline $\mathrm{pH}=6$ & $16.93 \pm 0.60$ & $3.16 \pm 0.17$ & $18.66 \pm 1.30$ & $0.339 \pm 0.012$ & $0.063 \pm 0.003$ & $2-3$ \\
\hline $\mathrm{pH}=7$ & $27.48 \pm 1.18$ & $8.32 \pm 0.33$ & $30.28 \pm 1.34$ & $0.550 \pm 0.024$ & $0.166 \pm 0.007$ & 0 \\
\hline $\mathrm{pH}=8$ & $27.33 \pm 1.26$ & $8.27 \pm 0.26$ & $30.25 \pm 1.13$ & $0.547 \pm 0.025$ & $0.165 \pm 0.005$ & 0 \\
\hline $\mathrm{pH}=9$ & $26.94 \pm 1.30$ & $8.28 \pm 0.44$ & $30.72 \pm 1.11$ & $0.539 \pm 0.026$ & $0.166 \pm 0.009$ & 0 \\
\hline $\mathrm{pH}=10$ & $23.65 \pm 1.29$ & $5.33 \pm 0.22$ & $22.53 \pm 1.42$ & $0.473 \pm 0.026$ & $0.107 \pm 0.004$ & $1-3$ \\
\hline
\end{tabular}

All the results presented are the mean values \pm SD for three independent replicates

N.D., not detected; $Y_{\mathrm{X} / \mathrm{s}}$, growth yield coefficient, g DCW/g VFAs, $Y_{\mathrm{L} / \mathrm{s}}$ lipid yield coefficient, $\mathrm{g}$ lipid/g VFAs

a Lag phase less than $3 \mathrm{~h}$ was recorded as 0 day

VFA system. The utilization of different single VFAs by yeasts would be interactively influenced in a mixed VFA system. Some researchers reported that different types of acids could be used simultaneously [19], while some also found yeasts would preferentially utilize acetic acid followed by propionic and butyric acid [36]. It was found in our previous study [17] that the utilization of different acids by $Y$. lipolytica in a mixed VFA system occurs in a step-wise manner, not in a synchronized manner. $Y$. lipolytica first uses acetic acid for lipid production. The metabolism of propionate and butyrate by $Y$. lipolytica is likely suppressed or halted when sufficient acetate is available as a carbon source. Therefore, acetic acid in VFA mixtures, which did not reach lethal concentration, could be metabolized first to obtain a certain level of cell density, thus further promoting the follow-up metabolism of residual VFAs. Moreover, different acids as carbon sources can induce general and acid-specific responses of yeast and increase phenotypic cell-to-cell heterogeneity [30]. Consequently, the likelihood that cells asynchronously resume growth during the acid-adaptation phase is increased, possibly contributing to a stronger acid tolerance of $Y$. lipolytica and a shorter lag phase than that of cultures on a single type of VFA. Meanwhile, the slightly lower biomass production on mixed VFAs than single acid could also be explained by the increase in phenotypic cell-to-cell heterogeneity, as it could be a result of the accumulation of cells that persisted in a viable but non-proliferating state.

\section{Biomass and lipid production of $Y$. lipolytica on FW and FVW fermentate that contained VFAs under alkaline conditions}

With a high soluble organic matter content, FW and FVW are easily degraded and can rapidly produce large amounts of VFAs during hydrolysis and acidification. Table 3 shows the characteristics of FW and FVW fermentate used by $Y$. lipolytica as feedstock for lipid production. As demonstrated in Table 4, the cultures with FW and FVW fermentates with an initial $\mathrm{pH}$ of 6.0 had a 48-72 h lag phase prior to an effective cell growth and resulted in an inferior performance on biomass and lipid production. Moreover, the VFAs in FW fermentate could not be fully consumed. These results indicated that the viability and vitality of yeasts were inhibited in this initial acidic environment. By increasing the initial $\mathrm{pH}$ to 7 and 8 , the yeasts could start proliferating with almost no lag phase $(<3 \mathrm{~h})$ and completely utilize VFAs. The biomass and lipid production were also significantly improved. The maximum lipid production of both cultures on FW 
Table 3 Characteristics of the supernatant of FW and FVW fermentate using as the feedstock for lipid production by Y. lipolytica

\begin{tabular}{lrr}
\hline Item & \multicolumn{2}{l}{ Concentration $\mathbf{( g / L )}$} \\
\cline { 2 - 3 } & \multicolumn{1}{c}{ FW } & \multicolumn{1}{c}{ FVW } \\
\hline SCOD & $56.76 \pm 2.22$ & $25.93 \pm 1.59$ \\
Total nitrogen & $2.14 \pm 0.08$ & $0.32 \pm 0.03$ \\
Total VFA & $35.35 \pm 1.45$ & $22.18 \pm 1.07$ \\
Acetic acid & $16.11 \pm 1.02$ & $5.34 \pm 0.87$ \\
Propionic acid & $6.69 \pm 0.55$ & $1.02 \pm 0.30$ \\
Butyric acid & $10.75 \pm 0.20$ & $14.23 \pm 0.42$ \\
Valeric acid & $0.83 \pm 0.06$ & $0.92 \pm 0.05$ \\
Isobutyric acid & $0.35 \pm 0.03$ & $0.21 \pm 0.02$ \\
Isovaleric acid & $0.62 \pm 0.02$ & $0.46 \pm 0.02$ \\
\hline
\end{tabular}

The concentration of the carbon and nitrogen sources in the effluent were measured after the solid content was removed by centrifugation and filtration. Three samples from the same experiment were analyzed

and FVW fermentates were obtained at an initial $\mathrm{pH}$ of 8 , indicating that this initial alkaline environment was also beneficial to cultures with organic waste-derived VFAs. The highest biomass and lipid production in the cultures with FW fermentate reached $14.65 \mathrm{~g} / \mathrm{L}\left(Y_{\mathrm{X} / \mathrm{S}}: 0.414\right)$ and $3.20 \mathrm{~g} / \mathrm{L}\left(Y_{\mathrm{L} / \mathrm{S}}: 0.091\right)$ with a lipid content of $21.86 \%$, respectively. Conversely, the highest biomass and lipid production in the cultures with FVW fermentate were $11.84 \mathrm{~g} / \mathrm{L}\left(Y_{\mathrm{X} / \mathrm{S}}: 0.534\right) 3.08 \mathrm{~g} / \mathrm{L}\left(Y_{\mathrm{L} / \mathrm{S}}: 0.139\right)$ with a lipid content of $26.02 \%$, respectively.

In contrast to FVW fermentate, FW fermentate with an initial $\mathrm{pH}$ of 8 did not show evident advantages over cultures with an initial $\mathrm{pH}$ of 7 . This result was probably due to the much higher nitrogen concentration of FW fermentate (TN: $2.14 \mathrm{~g} / \mathrm{L})$ than that of FVW fermentate (TN: $0.32 \mathrm{~g} / \mathrm{L}$ ). With an enhanced alkaline environment, ammonia-nitrogen tended to exist in the form of free ammonia, which could permeate into cells and induce stress on cell metabolism at high concentrations. The comparison of the cultures with FW and
FVW fermentates revealed that the lag phase duration of the cultures with FW fermentate at $\mathrm{pH} 6.0$ was shorter than that of the cultures with FVW fermentate, although the VFA concentration of FW fermentate (total VFAs: $35.35 \mathrm{~g} / \mathrm{L}$ ) was much higher than that of FVW fermentate (total VFAs: $22.18 \mathrm{~g} / \mathrm{L}$ ). This result could also be ascribed to the higher nitrogen concentration in the FW fermentate than in the FVW fermentate. In general, sufficient nitrogen is beneficial to yeast cell proliferation and growth. However, microbial lipids always accumulate under nitrogen-starved conditions [37]. $\mathrm{C} / \mathrm{N}$ has a critical effect on lipogenesis [38]. Oleaginous yeasts have difficulty in yielding a high lipid content at an initial $\mathrm{C} / \mathrm{N}$ ratio lower than $20 / 1$, and $\mathrm{C} / \mathrm{N}$ ratios from $40 / 1$ to $80 / 1$ generally seem to be appropriate for lipid accumulation in most oleaginous microorganisms [39]. With an initial $\mathrm{C} / \mathrm{N}$ of 7.76 , the cultures with FW fermentate had much lower lipid contents and $Y_{\mathrm{L} / \mathrm{S}}$ than those with FVW fermentate with an initial $\mathrm{C} / \mathrm{N}$ of 35.38 . In addition, the presence of unknown inhibitors in FW fermentate due to its complex composition may also adversely affect yeast lipogenesis [17].

\section{Fatty acid compositional analysis of lipids}

The fatty acid composition of lipids obtained during the batch cultivation of $Y$. lipolytica with high acetic acid concentrations induced by an alkaline $\mathrm{pH}$ of 8 and those with low acetic acid concentrations at $\mathrm{pH}$ of 6 was determined through GC analysis after esterification. The results are illustrated in Table 5. On the one hand, the accumulated fatty acids of the lipids in both cases were predominant with carbon chain lengths of $\mathrm{C} 16$ and $\mathrm{C} 18$, corresponding to those of vegetable and soybean oil [40]. On the other hand, the fatty acids of the lipids obtained in both cases are mostly unsaturated, with oleic acid (C18:1) acting as the major component, which is highly suitable for biodiesel production [17]. Although the ratio of $\mathrm{C} 16-\mathrm{C} 18$ of fatty acids obtained under alkaline conditions with high-content acetic acid decreased to $80.62 \%$

Table 4 Biomass and lipid production of Y. lipolytica on FW and FVW fermentate at different pH conditions

\begin{tabular}{|c|c|c|c|c|c|c|}
\hline \multirow{2}{*}{$\begin{array}{l}\text { Carbon source } \\
\text { Initial pH }\end{array}$} & \multicolumn{3}{|c|}{ VFA from FW fermentate } & \multicolumn{3}{|c|}{ VFA from FVW fermentate } \\
\hline & 6.0 & 7.0 & 8.0 & 6.0 & 7.0 & 8.0 \\
\hline VFA utilization ratio & $79.80 \%$ & $100 \%$ & $100 \%$ & $100 \%$ & $100 \%$ & $100 \%$ \\
\hline $\mathrm{DCW}(\mathrm{g} / \mathrm{L})$ & $9.12 \pm 0.59$ & $14.23 \pm 0.77$ & $14.65 \pm 0.55$ & $6.77 \pm 0.42$ & $10.52 \pm 0.50$ & $11.84 \pm 0.63$ \\
\hline Lipid conc. (g/L) & $1.35 \pm 0.14$ & $3.06 \pm 0.40$ & $3.20 \pm 0.37$ & $1.19 \pm 0.11$ & $2.77 \pm 0.28$ & $3.08 \pm 0.28$ \\
\hline Lipid content (wt\%) & $14.78 \pm 1.73$ & $21.52 \pm 1.51$ & $21.86 \pm 1.45$ & $17.58 \pm 1.16$ & $26.33 \pm 0.92$ & $26.02 \pm 1.04$ \\
\hline$Y_{X / S}$ & $0.323 \pm 0.020$ & $0.403 \pm 0.021$ & $0.414 \pm 0.015$ & $0.305 \pm 0.018$ & $0.474 \pm 0.023$ & $0.534 \pm 0.028$ \\
\hline$Y_{L / S}$ & $0.048 \pm 0.005$ & $0.087 \pm 0.011$ & $0.091 \pm 0.010$ & $0.054 \pm 0.005$ & $0.125 \pm 0.012$ & $0.139 \pm 0.012$ \\
\hline Lag phase (d) & $2-3$ & 0 & 0 & 3 & 0 & 0 \\
\hline
\end{tabular}

${ }^{a}$ Lag phase less than $3 \mathrm{~h}$ was recorded as 0 day 
Table 5 Main fatty acid composition of the lipids produced by $Y$. lipolytica under different culture conditions

\begin{tabular}{lll}
\hline Relative fatty acid content (\%) & \multicolumn{2}{c}{ Carbon source and initial pH } \\
\cline { 2 - 3 } & $\begin{array}{l}\mathbf{5 0} \mathbf{~ g / L} \text { acetic acid } \\
\text { at } \mathbf{~} \mathbf{~ 8 . 0}\end{array}$ & $\begin{array}{l}\mathbf{3} \mathbf{~ g / ~} \mathbf{~ a c e t i c} \\
\text { acid at pH } \\
\mathbf{6 . 0}\end{array}$ \\
\hline Total C16 and C18 & 80.62 & 89.26 \\
Unsaturated C16-C18 & 60.61 & 71.87 \\
Palmitic acid (C16:0) & 10.37 & 13.18 \\
Palmitoleic acid (C16:1) & 14.81 & 8.55 \\
Stearic acid (C18:0) & 9.64 & 4.21 \\
Oleic acid (C18:1) & 37.11 & 40.82 \\
Linoleic acid (C18:2) & 8.36 & 22.5 \\
Lionlenic acid (C18:3) & 0.33 & N.D. \\
Total C15 and C17 & 6.05 & 0.95 \\
Ginkgolic acid (C15:0) & 0.76 & N.D. \\
Ginkgolic acid (C15:1) & 3.31 & N.D. \\
Ginkgolic acid (C17:0) & 0.21 & N.D. \\
Ginkgolic acid (C17:1) & 1.77 & N.D. \\
\hline
\end{tabular}

Some other fatty acids ( $\mathrm{C} 14, \mathrm{C} 2 \mathrm{O}, \mathrm{C} 22)$ were also detected in trace amount and were not included in this table

compared with that in the cultures with low-content acetic acid at $\mathrm{pH} 6$, the fatty acid composition had a wider variety. In particular, the content of odd-numbered fatty acids $(\mathrm{C} 15, \mathrm{C} 17)$ slightly increased. The produced oddnumbered fatty acids can be applied to cosmetics, pesticide formulation, health care, and other aspects and thus have increased potential for various applications [41].

\section{Conclusions}

In contrast to cultures with low concentrations $(<10 \mathrm{~g} / \mathrm{L})$ of VFA, when high concentrations of VFAs as the carbon source were used, the adverse effects of VFAs ascribed to uncoupling became the dominating inhibitory factor. Thus, the slightly acidic conditions ( $\mathrm{pH}$ 5.6-7), which were considered the most favorable to oleaginous yeast cultivation, were inappropriate for cultures with a highcontent VFA because it could markedly aggravate inhibition caused by the unionized form of VFAs.

This study assumed and verified that alkaline conditions (pH 7-9) could effectively alleviate the lethal effect of high-content VFAs on $Y$. lipolytic to achieve a high cell-density and high lipid yield. An initial $\mathrm{pH}$ of 8 was determined as the optimal $\mathrm{pH}$ condition for the bioconversion of high-content VFAs to lipids by $Y$. lipolytica. Under this alkaline condition, cultures with high-content butyric acid could have comparable biomass and lipid production with cultures with acetic acid, whereas the performance with propionic acid was much inferior to that of the other acids. Mixed VFAs were more beneficial to fast adaptation and lipid production than single types of VFAs. Furthermore, feasibilities on undiluted FW and FVW fermentate were evaluated, and meaningful information was provided for practical application. In summary, this study could provide an effective strategy and useful information for the efficient bioconversion of high-content waste-derived VFAs into microbial lipids.

\section{Materials and methods}

Strain and inoculum preparation

Yarrowia lipolytica (CICC 31596) was obtained from the China Center of Industrial Culture Collection. For strain preservation, the yeast was maintained at $4{ }^{\circ} \mathrm{C}$ on YPD agar slants (2 mass\% agar powder) and subcultured monthly. The YPD medium contained $20 \mathrm{~g} / \mathrm{L}$ glucose, $20 \mathrm{~g} / \mathrm{L}$ peptone, and $10 \mathrm{~g} / \mathrm{L}$ yeast extract.

For the pre-culture of the seed cells, a loopfull of yeast cells were inoculated in $100 \mathrm{~mL}$ of YPD medium in a $250 \mathrm{~mL}$ flask and then incubated in a rotary shaker set to $180 \mathrm{rpm}$ at $28^{\circ} \mathrm{C}$ for $24 \mathrm{~h}$. The resulting cultures were re-inoculated in the YPD medium at a ratio of $10 \%(\mathrm{v} / \mathrm{v})$ and cultivated under the same conditions for $24 \mathrm{~h}$ as the seed culture.

\section{Culture conditions \\ Cultures on synthetic VFAs}

Batch culture experiments were performed in $100 \mathrm{~mL}$ of fermentation medium in $250 \mathrm{~mL}$ Erlenmeyer flasks. The nutrient medium used for all the cultures contained (g/L): $\mathrm{KH}_{2} \mathrm{PO}_{4}, 3 ; \mathrm{NH}_{4} \mathrm{Cl}, 1 ; \mathrm{MgSO}_{4} 7 \mathrm{H}_{2} \mathrm{O}, 1 ; \mathrm{FeCl}_{3} \cdot 6 \mathrm{H}_{2} \mathrm{O}$, $0.015 ; \mathrm{ZnSO}_{4} \cdot 7 \mathrm{H}_{2} \mathrm{O}, 0.0075$; and $\mathrm{CuSO}_{4} \cdot 5 \mathrm{H}_{2} \mathrm{O}, 0.0005$. In addition to the nutrient medium, acetic acid, butyric acid, propionic acid, and a mixture of these VFAs were used as sole carbon sources. The seed culture medium was inoculated in the fermentation medium at a ratio of $10 \%(\mathrm{v} / \mathrm{v})$. The $\mathrm{pH}$ of the fermentation medium was adjusted with $2 \mathrm{~mol} / \mathrm{L} \mathrm{NaOH}$ and $\mathrm{HCl}$ solutions when needed.

\section{Cultures on FW and FVW fermentate}

The anaerobic fermentation of FW and FVW was performed in an anaerobic digestion reactor at a working volume of $1.5 \mathrm{~L}$. FW was derived from the Dongcun solid waste treatment plant, which collects food waste from restaurants in Beijing, to prepare the feed. FVWs were collected from a market in the University of Science and Technology Beijing. Material processing and anaerobic fermentation were performed in accordance with a previous method [17].

After 5 days of fermentation, when hydrolysis and acidogenesis were finished, the effluent was collected and centrifuged at $5000 \mathrm{rpm}$ for $10 \mathrm{~min}$ to remove the suspended solids. The liquid supernatant was filtered through a $0.45 \mu \mathrm{m}$ membrane. The supernatant of FW and FVW fermentation effluent was then used as the 
feedstock without dilution or any additional nutrients for lipid production by $Y$. lipolytica. The culture was conducted in $250 \mathrm{~mL}$ Erlenmeyer flasks that contained $100 \mathrm{~mL}$ of medium at an initial $\mathrm{pH}$ of 6.0, 7.0, and 8.0.

The equipment and medium used in this study were steam autoclaved at $121{ }^{\circ} \mathrm{C}$ for $20 \mathrm{~min}$ before inoculation. All batch cultures were incubated in a rotary shaker at $180 \mathrm{rpm}$ and $28{ }^{\circ} \mathrm{C}$. This procedure was performed in triplicate.

\section{Analytical methods \\ Analysis of FW and FVW fermentate}

For FW and FVW fermentation, TS, VS, and $\mathrm{pH}$ were measured in accordance with the standard method [42]. Suspended solids were separated from the fermentation effluent via centrifugation at $5000 \mathrm{rpm}$ for $10 \mathrm{~min}$. The liquid supernatant was filtered through a $0.45 \mu \mathrm{m}$ membrane. Soluble COD (SCOD), total nitrogen (TN), and VFAs were then measured. SCOD and TN were analyzed via the $\mathrm{HACH}$ method. VFAs were determined as described in "VFA utilization" section.

\section{Cell growth and biomass production}

During cultivation, cell concentration was determined as the absorbance of the culture broth at $600 \mathrm{~nm}\left(\mathrm{OD}_{600}\right)$ to describe the cell growth curve. At the end of the stationary phase, biomass was harvested for lipid extraction and the corresponding measurements.

Dry cell weight $(\mathrm{DCW})$ was determined to describe biomass production. A $10 \mathrm{~mL}$ culture broth sample was centrifuged at $8000 \mathrm{rpm}$ for $10 \mathrm{~min}$. The cell pellet was washed twice with distilled water, dried to constant weight in an oven at $105^{\circ} \mathrm{C}$, and weighed [43].

\section{VFA utilization}

After the culture broth was centrifuged, the residual VFAs in the liquid supernatant were determined to describe the utilization of VFAs by the yeast. First, the liquid supernatant was filtered through a $0.45 \mu \mathrm{m}$ membrane. The concentrations of VFAs were then analyzed with a gas chromatograph (Shimadzu, GC-2014) fitted with a capillary column (Stabilwax-DA, $30 \mathrm{~m} \times 0.25 \mathrm{~mm} \times 0.25 \mu \mathrm{m}$ ) and a flame ionization detector. The temperature program was the same as that of a previous study [44].

\section{Lipid extraction and analysis}

Lipids were extracted in accordance with an adaptation [45] of the method of Bligh and Dyer [43]. Lipids were extracted from lyophilized biomass with chloroform/methanol $(2: 1 \mathrm{v} / \mathrm{v})$. The extracts were centrifuged at $5000 \mathrm{rpm}$ for 25 min to completely dissolve the lipids in the organic phase. The organic phase was sucked out and washed twice by using the same volume of $0.15 \%(\mathrm{w} / \mathrm{v}) \mathrm{NaCl}$ solution. Lipids were obtained after the purified chloroform layer was evaporated in a speed vacuum at $40^{\circ} \mathrm{C}$ until a constant weight was achieved.

Lipid composition was determined through the gas chromatography (GC) analysis of fatty acid methyl esters (FAMEs). The method of FAME preparation and GC analysis are described in a previous study [17].

\section{Confocal fluorescence microscopy analysis}

Confocal fluorescence microscopy was carried out to illustrate the cell growth and lipid accumulation of Y. lipolytica. Approximately $10 \mu \mathrm{L}$ of the cell suspension sample was collected from cultures at the stationary phase, subsequently stained with $10 \mu \mathrm{L}$ of Nile red, and imaged with a laser scanning confocal fluorescence microscope (Leica TCS SP2) under an excitation wavelength of $543 \mathrm{~nm}$.

\section{Abbreviations}

VFA: volatile fatty acids; DCW: dry cell weight; $Y_{x / s}$ : growth yield coefficient, g DCW/g VFAs; $Y_{\text {Ls: }}$ : lipid yield coefficient, g lipid/g VFAs; TS: total solid; VS: volatile solid; rpm: rotations per minute; YPD: yeast peptone dextrose; FW: food waste; FVW: fruit and vegetable waste; N.D.: not detected.

\section{Acknowledgements}

The authors acknowledged the financial support by the Bill \& Melinda Gates Foundation. The authors would also like to thank the National Environment and Energy International Cooperation Base for their support.

\section{Authors' contributions}

RLG designed the study, performed the experiments, analyzed the results and drafted the manuscript. ZFL, as the corresponding author, developed the concept of this research, supervised the progress of this research, and commented on the manuscript. WJB assisted in the experiments. XQZ, LZ and SKC participated in the design of the study and commented on the manuscript. All authors read and approved the final manuscript.

Funding

This study is funded by the Bill \& Melinda Gates Foundation (Global Development Grant No. OPP1051913).

\section{Availability of data and materials}

All data and material used in the current study are available from the corresponding author on reasonable request.

Ethical approval and consent to participate Not applicable.

Consent for publication

All authors give consent to publish the research in Biotechnology for Biofuels.

Competing interests

The authors declare that they have no competing interests.

Received: 7 December 2018 Accepted: 27 December 2019

Published online: 06 January 2020 


\section{References}

1. Huang XF, Shen Y, Luo HJ, Liu JN, Liu J. Enhancement of extracellular lipid production by oleaginous yeast through preculture and sequencing batch culture strategy with acetic acid. Bioresour Technol. 2018;247:395-401.

2. Demirbas A. Political, economic and environmental impacts of biofuels: a review. Appl Energy. 2009;86(Suppl):108-17.

3. Liu NJ, Huang XF, Chen R, Yuan M, Liu J. Efficient biocoversion of highcontent volatile fatty acids into microbial lipids by Cryprococcus curvatus ATCC 20509. Bioresour Technol. 2017:239:394-401.

4. Fei Q, Chang HN, Shang L, Choi JD, Kim N, Kang J. The effect of volatile fatty acids as a sole carbon source on lipid accumulation by Cryptococcus albidus for biodiesel production. Bioresour Technol. 2011;102:2695-701.

5. Gong ZW, Zhou WT, Shen HW, Yang ZH, Wang GH, Zuo ZY, Hou YL, Zhao ZBK. Co-fermentation of acetate and sugars facilitating microbial lipid production on acetate-rich biomass hydrolysates. Bioresour Technol. 2016;207:102-8

6. Lian J, Garcia-Perez M, Coates R, Wu H, Chen S. Yeast fermentation of carboxylic acids obtained from pyrolytic aqueous phases for lipid production. Bioresour Technol. 2012;118:177-86.

7. Zheng Y, Chi Z, Ahring BK, Chen S. Oleaginous yeast Cryptococcus curvatus for biofuel production: ammonia's effect. Biomass Bioenergy. 2012;37:114-21.

8. Chang HN, Kim NJ, Kang J, Jeong CM. Biomass-derived volatile fatty acid platform for fuels and chemicals. Biotechnol Bioprocess Eng. 2010;15:1-10.

9. Ma H, Chen X, Liu H, Liu H, Fu B. Improved volatile fatty acids anaerobic production from waste activated sludge by $\mathrm{pH}$ regulation: alkaline or neutral pH? Waste Manage. 2015:48:397-403.

10. Yin J, Wang K, Yang Y, Shen D, Wang M, Han M. Improving production of volatile fatty acids from food waste fermentation by hydrothermal pretreatment. Bioresour Technol. 2014;171:323-9.

11. Zhang D, Li X, Jia S, Dai L, Zhao J, Chen Y, Dai X. A review: factors affecting excess sludge anaerobic digestion for volatile fatty acids production. Water Sci Technol. 2015;72:678-88.

12. Yuan Q, Sparling R, Oleszkiewicz JA. VFA generation from waste activated sludge: effect of temperature and mixing. Chemosphere. 2011:82:603-7.

13. Khiewwijit R, Temmink H, Labanda A, Rijnaarts $H$, Keesman KJ. Production of volatile fatty acids from sewage organic matter by combined bioflocculation and alkaline fermentation. Bioresour Technol. 2015;197:295-301.

14. Liu J, Yuan M, Liu JN, Huang XF. Biocoversion of mixed volatile fatty acids into microbial lipids by Cryprococcus curvatus ATCC 20509. Bioresour Technol. 2017;241:645-51.

15. Rodrigues $G$, Pais $C$. The influence of acetic and other weak carboxylic acids on growth and cellular death of the yeast Yarrowia lipolytica. Food Technol Biotechnol. 2000:38(1):27-32.

16. Fontanille P, Kumar V, Christophe G, Nouaille R, Larroche C. Bioconversion of volatile fatty acids into lipids by the oleaginous yeast Yarrowia lipolytica. Bioresour Technol. 2012:114:443-9.

17. Gao RL, Li ZF, Zhou XQ, Cheng SK, Zheng L. Oleaginous yeast Yarrowia lipolytica culture with synthetic and food waste-derived volatile fatty acids for lipid production. Biotechnol Biofuels. 2017;10:247-62.

18. Vanessa B, Laurent P, Gwendoline C, Andre L, Christian L, Pierre F. Improvement and modeling of culture parameters to enhance biomass and lipid production by the oleaginous yeast Cryptococcus curvatus grown on acetate. Bioresour Technol. 2015;192:582-91.

19. Xu X, Kim JY, Cho HU, Park HR, Park JM. Bioconversion of volatile fatty acids from macroalgae fermentation into microbial lipids by oleaginous yeast. Chem Eng J. 2015;264:735-43.

20. Liu J, Yuan M, Liu JN, Lu L, Peng KM, Huang XF. Microbial conversion of mixed volatile fatty acids into microbial lipids by squencing batch culture strategy. Bioresour Technol. 2016:222:75-81.

21. Gong Z, Shen H, Zhou W, Wang Y, Yang X, Zhao ZK. Efficient conversion of acetate into lipids by the oleaginous yeast Cryptococcus curvatus. Biotechnol Biofuels. 2015;8(1):189-98.

22. Christophe G, Lara DJ, Kumar V, Nouaille R, Fontanille P, Larroche C. Production of oils from acetic acid by the oleaginous yeast Cryptococcus curvatus. Appl Biochem Biotechnol. 2012;167:1270-9.

23. Verduyn C, Postma E, Scheffers WA, Van Dijken JP. Effect of benzoic acid on metabolic fulxes in yeasts: a continuous-culture study on the regulation of respiration and alcoholic fermentation. Yeast. 1992;8(7):501-17.
24. Cherrington CA, Hinton M, Chopra I. Effect of short-chain organic acids on macromolecular synthesis in Escherichia coli. J Bacteriol. 1990;68:69-74.

25. Abbott DA, Suir E, Van Maris AJA, Pronk JT. Physiological and transcriptional responses to high concentrations of lactic acid in anaerobic chemostat cultures of Saccharomyces cerevisiae. Appl Environ Microbiol. 2008;74:5759-68.

26. Ratledge C. Microorganisms for lipids. Acta Biotechnol. 1991;11:429-38.

27. Chi ZY, Zheng YB, Ma JW, Chen SL. Oleaginous yeast Cryptococcus curvatus culture with dark fermentation hydrogen production effluent as feedstock for microbial lipid production. Int J Hydrogen Energy. 2011;36:9542-50

28. Zheng YB, Li TT, Yu XC, Bates PD, Dong T, Chen S. High-density fed-batch culture of a thermotolerant microalga Chlorella sorokiniana for biofuel production. Appl Energy. 2013;108:281-7.

29. Magdalena R, Zbigniew L, Thierry D, Patrick F, Jean MN. Lipid production by the oleaginous yeast Yarrowia lipolytica using industrial by-products under different culture conditions. Biotechnol Biofuels. 2015;8:104-14.

30. Guo ZP, Olsson L. Physiological responses to acid stress by Saccharomyces cerevisiae when applying high initial cell density. FEMS Yeast Res. 2016. https //doi.org/10.1093/femsyr/fow072.

31. Holyoak CD, Stratford M, McMullin Z, et al. Activity of the plasma membrane $\mathrm{H}$-ATPase and optimal glycolytic flux are required for rapid adaptation and growth of Saccharomyces cerevisiae in the presence of the weak-acid preservative sorbic acid. Appl Environ Microbiol. 1996;62(9):3158-64.

32. Baik HS, Bearson S, Dunbar S, Foster JW. The acid tolerance of Salmonella typhimurium provides protection against organic acids. Microbiology. 1996:142:3195-200.

33. Royce LA, Liu P, Stebbins MJ, Hanson BC, Jarboe LR. The damaging effects of short chain fatty acids on Escherichia coli membranes. Appl Microbiol Biothchnol. 2013;97:8317-27.

34. Santamauro F, Whiffin FM, Scott RJ, Chuck CJ. Low-cost lipid production by an oleaginous yeast cultured in non-sterile conditions using model waste resources. Biotechnol Biofuels. 2014;7:34-45.

35. Fradinho J, Oehmen A, Reis MAM. Photosynthetic mixed culture polyhydroxyalkanoate (PHA) production from individual and mixed volatile fatty acids (VFAs): substrate preferences and co-substrate uptake. J Biotechnol. 2014;185:19-27.

36. Vajpeyi S, Chandran K. Microbial conversion of synthetic and food wastederived VFA to lipids. Bioresour Technol. 2015;188:49-55.

37. Papanikolaou S, Aggelis G. Lipids of oleaginous yeasts. Part II: technology and potential applications. Eur J Lipid Sci Technol. 2011;113:1052-73.

38. Ratledge C. Regulation of lipid accumulation in oleaginous micro-organisms. Biochem Soc Trans. 2002;30:1047-9.

39. Seo Y, Lee I, Han J. Cultivation and lipid production of yeast Cryptococcus curvatus using pretreated waste active sludge supernatant. Bioresour Technol. 2013;135:304-8.

40. Zlatanov M, Pavlova K, Antova G, Angelova-Romova M, Georgieva K, Rousenova-Videva S. Biomass production by antarctic yeast strains: an investigation on the lipid composition. Biotechnol Biotechnol Equip. 2014;24:2096-101.

41. Rezanka T, Kolouchovaa I, Sigler K. Precursor directed biosynthesis of oddnumbered fatty acids by different yeasts. Folia Microbiol. 2015;60:457-64.

42. APHA. Standard Methods for the Examination of Water and Wastewater 21sted. American Public Health Association, American Water Works Association, Water Environment Federation, Washington, DC. 2005.

43. Bligh E, Dyer WJ. A rapid method of total lipid extraction and purification. Can J Biochem Physiol. 1959;37:911-7.

44. Jiang J, Gong C, Wang J, Tian S, Zhang Y. Effects of ultrasound pre-treatment on the amount of dissolved organic matter extracted from food waste. Bioresour Technol. 2014:155:266-71.

45. Bourque SD, Titorenko VI. A quantitative assessment of the yeast lipidome using electrospray ionization mass spectrometry. J Vis Exp JoVE. 2009. https ://doi.org/10.3791/1513

\section{Publisher's Note}

Springer Nature remains neutral with regard to jurisdictional claims in published maps and institutional affiliations. 